NBER WORKING PAPER SERIES

\title{
EXPECTATIONS-BASED LOSS AVERSION MAY HELP EXPLAIN SEEMINGLY DOMINATED CHOICES IN STRATEGY-PROOF MECHANISMS
}

\author{
Bnaya Dreyfuss \\ Ori Heffetz \\ Matthew Rabin \\ Working Paper 26394 \\ http://www.nber.org/papers/w26394 \\ NATIONAL BUREAU OF ECONOMIC RESEARCH \\ 1050 Massachusetts Avenue \\ Cambridge, MA 02138 \\ October 2019
}

A version of this paper constituted Dreyfuss's MA thesis at The Hebrew University of Jerusalem. We thank Ned Augenblick, Alon Eizenberg, Zoë Hitzig, Shengwu Li, Alex Rees-Jones, Assaf Romm, Charlie Sprenger, the JESC-lab group and especially Matan Gibson, Ofer Glicksohn, Guy Ishai and Kobi Mizrahi, and seminar/conference participants at the Hebrew University, UCSB, University of Utah, UC Berkeley, and BEAM for invaluable comments. This research project was supported by the I-CORE program of the Planning and Budgeting Committee and the Israel Science Foundation (grant no. 1821/12). The views expressed herein are those of the authors and do not necessarily reflect the views of the National Bureau of Economic Research

NBER working papers are circulated for discussion and comment purposes. They have not been peer-reviewed or been subject to the review by the NBER Board of Directors that accompanies official NBER publications.

(C) 2019 by Bnaya Dreyfuss, Ori Heffetz, and Matthew Rabin. All rights reserved. Short sections of text, not to exceed two paragraphs, may be quoted without explicit permission provided that full credit, including $\odot$ notice, is given to the source. 
Expectations-Based Loss Aversion May Help Explain Seemingly Dominated Choices in Strategy-Proof Mechanisms

Bnaya Dreyfuss, Ori Heffetz, and Matthew Rabin

NBER Working Paper No. 26394

October 2019

JEL No. B49,D47,D82,D84,D91

\begin{abstract}
$\underline{\text { ABSTRACT }}$
Deferred Acceptance (DA), a widely implemented algorithm, is meant to improve allocations: under classical preferences, it induces preference-concordant rankings. However, recent evidence shows that - in both real, large-stakes applications and experiments-participants frequently play seemingly dominated, significantly costly, strategies that avoid small chances of good outcomes. We show theoretically why, with expectations-based loss aversion, this behavior may be partly intentional. Reanalyzing existing experimental data on random serial dictatorship (a restriction of DA), we show that such reference-dependent preferences, with a degree and distribution of loss aversion that explain common levels of risk aversion elsewhere, fit the data better than no-lossaversion preferences.

Bnaya Dreyfuss

Department of Economics

The Hebrew University of Jerusalem

Mt. Scopus

Jerusalem

Israel

bnaya3fuss@gmail.com

Ori Heffetz

S.C. Johnson Graduate School of Management

Cornell University

324 Sage Hall

Ithaca, NY 14853

and The Hebrew University of Jerusalem

and also NBER

oh33@cornell.edu

Matthew Rabin

Department of Economics

Littauer Center M-8

Harvard University

Cambridge, MA 02138

MatthewRabin@fas.harvard.edu
\end{abstract}


Blessed is he who expects nothing, for

he shall never be disappointed.

Alexander Pope

\section{Introduction}

Centralized clearinghouses are used across the world to match applicants to positions. A common example is assigning students to schools. The assignment is determined as a function of students' rankings of schools and some combination of schools' rankings of students and predetermined priorities. These clearinghouses are increasingly adopting strategy-proof (SP) mechanisms, in which, with standard utility, a ranking of alternatives that does not coincide with an applicant's true preferences is a (weakly) dominated strategy. One such mechanism that is particularly popular is the deferred-acceptance algorithm (Gale and Shapley, 1962 , henceforth DA) 1 $^{1}$

However, seemingly dominated rankings in DA-based allocation contexts are evident in a number of recent field and lab studies, reviewed in detail below. From the point of view of classical economic theory, the evidence is puzzling: in the lab, a substantial fraction of participants in simple allocation games submit lists that rank smaller amounts of money above larger ones; in real-world clearinghouses, supposedly well-informed school applicants do not rank a program that comes with a fellowship above the same program without the fellowship and, when such a ranking prompts a pop-up notification questioning their choice, they click OK to "keep the present preference ranking anyway."

This paper explores the idea that at least some apparently suboptimal behavior is in fact intentional behavior by individuals with expectations-based reference-dependent (EBRD) preferences. Intuitively, submission of a list creates expectations, and loss-averse individuals may want to avoid generating high expectations in order to avoid experiencing future disappointment. Specifically, an individual who is loss-averse around her endogenous expectation-based reference point may submit a list with a lower-value position (or a smaller sum of money) that she expects to get, above a higher-value position (or a larger sum) that she has little chance of getting. In the first part of the paper, using the framework of Köszegi and Rabin (2009), we show this theoretically, and derive specific empirical predictions. In the second part, reanalyzing experimental data from $\mathrm{Li}(2017)$, we show that the EBRD model fits the data substantially better than the no-loss-aversion, standard model.

Within the literature, a ranking that coincides with (true) preferences is referred to as

\footnotetext{
${ }^{1}$ For example, DA-based mechanisms are used in school choice in the US (e.g. Abdulkadiroğlu et al. 2005), Israel (Hassidim et al., 2018), Hungary (Shorrer and Sóvágó, 2018), and Australia (Artemov et al. 2017). For a recent review of the use of DA-based mechanisms, see Fack et al. (2019).
} 
truthful, and one that does not coincide with preferences is referred to as a misrepresentation. We reluctantly keep using these terms, even though they are likely misnomers both from the EBRD-theory's perspective and from the point of view of those participanting in the mechanisms, especially since participants are not typically told that their ranking is a matter of honesty. We keep this terminology mainly for consistency with the literature, use it purely descriptively, and imply no normative interpretation.

Briefly, the DA mechanism works - and is typically communicated to applicants - as follows: "The process begins with an attempt to match an applicant to the program most preferred on that applicant's rank-order list (ROL). If the applicant cannot be matched to that first-choice program, an attempt then is made to place the applicant into the second-choice program, and so on, until the applicant obtains a tentative match or all the applicant's choices on the ROL have been exhausted. When all applicant rank-order lists have been considered, all tentative matches become final.'2 2 The tentativeness of matches, which is emphasized to participants, means that in each round, all yet-unmatched and all already(tentatively)-matched applicants to each program compete with each other anew. It is this feature of DA that guarantees that applicants cannot gain by misrepresenting their preferences. In contrast, the "Boston mechanism," explained in, e.g., Hakimov and Kübler (2019), is not SP. In that mechanism, all matches are final-hence it is sometimes referred to as the immediate-acceptance, rather than deferred-acceptance, algorithm. That "first come first served" algorithm is easy to manipulate: ranking a school higher than its value in order to increase its admission probability is in fact, in many cases, a best response.

Evidence of misrepresentation in DA settings is found in several lab experiments. In a typical experiment, participants submit ROLs of hypothetical positions that are assigned different amounts of real money (see Hakimov and Kübler, 2019 for a recent review). Chen and Sönmez (2006), Calsamiglia et al. (2010), Klijn et al. (2013), Ding and Schotter (2017), and $\operatorname{Li}(2017)$, for example, find that between 19 and 45 percent of submitted ROLs are not ranked monotonically by their monetary value. Notably, Rees-Jones and Skowronek (2018) conduct an online experiment with 1,714 medical students immediately after their participation in the National Resident Matching Program (NRMP), a much-studied example of a carefully designed strategy-proof market, and find that when placed in an analogous, incen-

\footnotetext{
${ }^{2}$ This text is copied (unedited, except for the addition of four hyphens) from the FAQ page on the website of the National Resident Matching Program (NRMP), which we briefly discuss below. The original text ends with a weblink to a "How the Matching Algorithm Works" page (http://www.nrmp.org/ matching-algorithm/), which contains a short animated video that explains the mechanism and demonstrates it with a simple example. The introduction to the video reads: "The NRMP uses a mathematical algorithm to place applicants into residency and fellowship positions. Research on the algorithm was the basis for awarding the 2012 Nobel Prize in Economic Sciences. To make the matching algorithm work best for you, create your rank order list in order of your true preferences, not how you think you will match."
} 
tivized matching task, 23 percent of participants misrepresent their preferences.3 In these experiments, applicants often seem to factor into their ranking the probability of admission on top of monetary value.

In contrast with these lab settings, where (because respondents are essentially asked to rank amounts of money) preferences are assumed to be known, in field settings identifying misrepresentation is harder, because applicants' preferences over programs are not generally known. In recent studies, however, researchers identified an important exception: in some DA-based allocation mechanisms, applicants submit ROLs that may include, as two separately ranked options, a funded position - e.g., a study program with a fellowship - and an identical non-funded position-i.e., the same program without the fellowship. This allows researchers to detect what they term obvious misrepresentations: ROLs where a funded position is not ranked above an identical non-funded one (Hassidim et al., 2018; Shorrer and Sóvágó, 2018; Artemov et al., 2017). Obvious misrepresentations are further categorized by researchers into obvious flippings, i.e., ROLs with a funded position ranked below an identical non-funded one, and obvious droppings, i.e., ROLs that include a non-funded position, but not an identical funded one 4

Like the misrepresentations found in the lab, the obvious misrepresentations identified in the field are prevalent. The studies above find that of the ROLs that contain at least one program that offers funding, between 19 and 35 percent obviously misrepresent. Generally, applicants who are less likely to receive funding (as determined by measures of ability and socioeconomic status) are found more likely to obviously misrepresent. While this finding may suggest that misrepresentation is common mainly when it is likely to be irrelevant, these misrepresentations are sometimes costly. For example, Shorrer and Sóvágó (2018) show that in the Hungarian college-admissions market 12-19 percent of the obvious misrepresentations are ex-post costly, with an average cost of $\$ 347-\$ 735$ per misrepresentation $(\$ 3,000-\$ 3,500$ per costly misrepresentation). Similar evidence has been found in Israel (2-8 percent of obvious misrepresentations are ex-post costly; Hassidim et al., 2018) and Australia (1-20 percent; Artemov et al., 2017). Of course, obvious misrepresentations are a lower bound; their prevalence in these markets raises the possibility of less-than-obvious misrepresentation as a potentially broader phenomenon in various DA-based markets.5

\footnotetext{
${ }^{3}$ Although the NRMP itself uses a modified version of DA that complicates the strategic incentives, simulations in Roth and Peranson (1999) suggest that effectively all students remain incentivized to truthfully reveal their preferences.

${ }^{4}$ Note, however, that an obvious flipping and an obvious dropping result in the same outcome. If the applicant is admitted to the non-funded program, then all the programs that she ranked below the nonfunded program become irrelevant. If she is rejected from the non-funded program, then she will also be rejected from an identical funded one.

${ }^{5}$ In addition to the above choice-based evidence of misrepresentation, there is also some survey-based
} 
In all the above studies, the authors offer explanations for costly preference misrepresentation. These fall into two main categories: incorrect beliefs and non-classical preferences. We start with the first. Extreme pessimism could lead applicants to incorrectly assign essentially zero probability to the event of getting funding, and so they simply do not bother ranking funded positions. Such over-pessimism seems less likely to explain results from the lab, where it is not clear what prevalent bias would generate that particular belief. Alternatively, applicants could fail to understand the mechanism's strategy-proofness. The error most plausible to explain the observed misrepresentation is a mistaken belief that higher ranking will increase the probability of admission - as is true for mechanisms such as the Boston mechanism discussed earlier. However, such a mistaken belief cannot explain obvious droppings. Moreover, in markets with admission-score cutoffs, where applicants are matched with their highest-ranked program whose (ex-post common-knowledge) cutoff does not exceed their (privately known) admission score (e.g., Artemov et al., 2017; Shorrer and Sóvágó, 2018), such a mistake would amount to believing that ranking a program higher could decrease its admission-score cutoff. In addition, this explanation appears implausible in settings where the information regarding strategy-proofness is made salient to applicants ${ }^{6}$

The second category of explanations is closer to the focus of our paper, as it concerns utility-maximizing behavior with correct beliefs but non-classical preferences. Ego utility (Köszegi, 2006), for example, can predict that applicants may choose to rank higher those schools at which they have higher chances of admission, in order to avoid learning that they would be rejected by more attractive schools. However, with cutoff scores, this explanation too seems implausible, because applicants generally cannot avoid the information regarding what would happen if their ROL were truthful. Altruistic motives can also explain why some applicants may omit funded positions. Finally, by not applying for funding, applicants may attempt to signal (including to themselves) pro-sociality, generosity, or wealth. While we believe that wrong beliefs, simple mistakes, and the above non-classical preferences may all play a role in explaining some of the misrepresentation, in the rest of this paper we explore evidence. Rees-Jones (2018) reports that out of 558 respondents surveyed after their NRMP submission, 17 percent self-assessed their submitted ROLs as non-truthful. However, this evidence is hard to interpret because in many cases respondents' verbal explanations suggest that their ROLs did truly represent some other notion of preferences, e.g., their family preferences - and should therefore not be considered here as evidence of misrepresentation. Hassidim et al. (2018) report that in two surveys conducted in 2014 and 2015, 18 percent of 367 respondents reported submitting a ROL that was only "partially truthful" (2014), and 20 percent of 292 respondents reported that their ROL ranked some program higher or lower relative to their true preferences (2015). In the 2015 survey, where respondents provide verbal explanations for this behavior, they often mention considerations of chances of admission (which may also suggest that in this case respondents do interpret the preferences question as intended).

${ }^{6}$ The FAQ section in Hassidim et al. (2018) explicitly advises applicants to rank truthfully. For experimental evidence on the effect of advice on behavior in a different strategy-proof mechanism (top trading cycles), see Guillen and Hing (2014) and Guillen and Hakimov (2018). 
the role that EBRD may play.

In section 1 we outline the EBRD model. Developed by Kőszegi and Rabin $(2006,2007$; 2009), the model builds on Prospect Theory (Kahneman and Tversky, 1979), and is meant to generalize and formalize its value function. (The ideas of framing, editing, and probability weighting are not included; in section 3.6 we discuss how including probability weighting may affect our predictions.) The model's two main purposes are, first, to explain how reference points affect behavior, i.e., how people react to various departures from a posited reference point, and, second, to define how said reference points are endogenously determined. Because different ROLs induce different expectations and hence different reference points, the utility function in the EBRD model is ROL-dependent: when the agent optimizes she takes into account the effect of the ROL on her expectations and hence on her reference point. Other models of disappointment aversion, such as Bell (1985), Loomes and Sugden (1986) and Gul (1991) have similar predictions. However, the last two do not predict obvious misrepresentations, as they preclude violations of first-order stochastic dominance.

In section 2 we derive predictions. First, we analyze a simple case where there are only two schools. We show that for loss-averse agents, there are plausible conditions where the utility-maximizing ROL differs from ranking by value, with either the order of schools flipped or a school omitted. Intuitively, an applicant who is likely to get matched with a school will feel a loss when matched with any other school (even a better one); this can create attachment to the high-probability school - an endowment effect for schools. By moving the (possibly less-preferred) school up on her ROL, and updating her reference point accordingly, she can reduce the chance of loss. In addition, avoiding high expectations of matching with other, possibly better schools reduces the chances of the painful loss of not matching with them. For agents with a high degree of loss aversion, when this latter effect is strong enough, it can even result in the omission of a school altogether (rather than just flipping the order of schools), violating first-order stochastic dominance (FOSD). We provide the most general result in subsection 2.5, where we show that the main two-schools result can be generalized to any number of schools. Specifically, we show that so long as the likelihood of a school's admission does not increase with the school's value, for plausible combinations of parameters misrepresentation always occurs. We close the section with a simple example that demonstrates the model's explanation of existing field evidence of obvious misrepresentation.

All else equal, the EBRD model predicts that some applicants with low chances of matching with funded positions - generally, weaker students - will prefer not to rank them above identical non-funded ones. As discussed above, this prediction seems to be consistent with existing empirical evidence. Of course, in the field, low probability of getting into a funded program may be highly correlated with low ability, and therefore may also be correlated 
with ability-related mistakes, such as misunderstanding of the strategy-proofness of the DA mechanism. However, Shorrer and Sóvágó (2018) exploit a reform that substantially increased the selectivity of admission with funding in some fields of study in order to show that lowering the probability of receiving funding causally increases the number of observed misrepresentations.

Naturally, with this field evidence alone, a theory in which applicants with classical preferences are more likely to make mistakes when they are less costly may be enough to explain the data. In section 3 we reanalyze the data from one of the experimental treatments in $\operatorname{Li}(2017)$, and test our EBRD explanation against such a benchmark ${ }^{7}$ In that treatment, over ten rounds, four different sums of money, between $\$ 0$ and $\$ 1.25$, are allocated to four participants using Random Serial Dictatorship (RSD) - a restriction of DA to one-sided markets with priorities. In RSD, players' ROLs are processed by a randomly-determined priority, and each player receives the highest-ranked prize on her ROL that is still left to choose from by the time her list is processed. Participants first learn their randomly drawn "priority score" - an integer between 1 and 10 that strongly signals their actual priorityand then rank the four monetary prizes in any order they choose. After describing the experimental setup, we present detailed theoretical predictions: for each possible choice situation, we show the expected EBRD utility from the submission of each possible ROL as a function of the degree of loss aversion. A loss-averse participant may rank high amounts of money lower when her priority score is lower.

Li (2017) finds that 29 percent of submitted ROLs are not ordered by value. We explore the extent to which the empirical misrepresentation patterns match our theoretical predictions. The distributions of ROLs conditional on different priority scores seem to fit the EBRD model's prediction: some subjects indeed seem to try to maximize their chances of winning their first ranked choice, even at the expense of reducing their expected monetary payoff. For example, as we show in table 3, as the ROL-submitter's priority score decreases, the percent of ROLs with prizes ranked by their value decreases steeply: it is at most 91, 79, and 61 percent, respectively, for subjects with the three highest, four middle, and three lowest priority scores. At the same time, the percent of ROLs with the lowest prize ranked first increases as priority decreases: it is up to 3, 11, and 21 percent for high-, middle-, and low-priority-score subjects, respectively. In between, a hump-shaped upper-bound of 13, 29, and 25 percent of ROLs, respectively, rank one of the two middle prizes on top of their list-consistent with the theory.

\footnotetext{
${ }^{7}$ We do not analyze data from Li's other DA-related treatment since there, the EBRD model's predictions coincide with those of the classical, reference-independent-preferences model. (These coinciding predictions are largely confirmed; see footnotes 22 and 27 below.) Li has additional treatments that involve auctions, which we do not explore in this paper.
} 
Our main empirical results are in subsection 3.4. We formally test the fit of the EBRD model to the data, comparing it to the fit of the no-loss-aversion alternative. We first use a logit, random-utility model (where choosers make randomly drawn mistakes, whose probability decreases with their cost) to estimate a single (population-wide) coefficient of loss aversion, $\lambda$. We estimate $\lambda=2.0$, with a standard error of 0.2 , so the data clearly favors $\lambda>1$ over the nested classical non-EBRD preferences, corresponding to $\lambda=1$. With a likelihood-ratio test statistic $=32(p$-value $<0.00001)$, the observed choices are more than $10^{7}$ times likelier under the $\lambda=2$ than the $\lambda=1$ model. This result passes several placebo/permutation tests, as the same equality-of-fit hypothesis is never rejected at such significance levels on synthetic, randomly generated datasets that we construct to match the real data on several dimensions. The result holds in both earlier and later rounds of the experiment, and is also replicated $(\lambda=2.2, \mathrm{SE}=0.4)$ on a second dataset: a one-shot (rather than a ten-round) version of the same experiment, with stakes that are 12 times higher, conducted by $\mathrm{Li}(2017)$ after the original experiment. Finally, we estimate both individual-level and (parametric) population-wide models of heterogeneity in the coefficient of loss aversion, and find mean $\lambda=1.6-3.0$, with substantial heterogeneity. We close section 3 with further evidence that Li's subjects did not misperceive the mechanism in the experiment to be the Boston mechanism, followed by a brief discussion of past experimental evidence of FOSD violations that can be explained by the EBRD model.

In section 4 we conclude, and discuss implications of our findings. Following our analysis, a seemingly dominated choice may not be a calculation mistake. But, if EBRD preferences themselves are mistaken with respect to some notion of agents' true preferences, then a seemingly dominated choice may still suggest a mistake of a different kind.

\section{A Model of News Utility}

We use a simplified version of Köszegi and Rabin's 2009) EBRD framework. Lori is a decision maker (DM) with EBRD preferences, about to submit her ROL of schools to a DA-based centralized clearinghouse.

In the general model, Lori lives $T+1$ periods. In each period, a $K$-dimensional consumption vector $\boldsymbol{c}_{\boldsymbol{t}}=\left(c_{t}^{1}, \ldots c_{t}^{K}\right)$ is realized. In our setting, we parse Lori's life into 3 periods, $t=1,2,3$. Lori consumes only in period 3 , and the only consumption dimensions are schooldetermined lifetime consumption - a separate dimension for each school - and, in the last example below, money. Since in many cases, matching with a specific school determines much of the rest of an applicant's lifetime experience-including one's social circle, economic status, friends, spouse, city of residence, etc.- the "consumption" of a specific school 
in our setting represents attending the school as well as enjoying the resulting flow of lifetime consumption. In period 1, Lori learns about the submission process, forms beliefs about her chances, and submits her ROL. In period 2, the uncertainty resolves and she learns about her match. In period 3, which represents the rest of Lori's life, she consumes. (We discuss this periodization in section 2.2.)

In the general model, Lori starts every period $t$ with beliefs she inherited from the previous period, denoted $F_{t-1}=\left.\left\{F_{t-1, \tau}\right\}\right|_{\tau=t} ^{T}$, where $F_{t, \tau}$ are the beliefs Lori holds in period $t$ regarding her consumption in period $\tau \geq t$ in each of the $K$ dimensions. Within each period, up to five things may happen: some of the uncertainty may resolve, Lori may take an action, further uncertainty may resolve, $\boldsymbol{c}_{\boldsymbol{t}}$ is consumed, and Lori updates her beliefs $F_{t}=\left.\left\{F_{t, \tau}\right\}\right|_{\tau=t} ^{T}$, where $F_{t, t}$ assign probability 1 to $\boldsymbol{c}_{\boldsymbol{t}}$.

In our setting, Lori takes one action: the submission of a ROL in period 1, which determines $F_{1,3}$, Lori's beliefs at the end of period 1 regarding her consumption in period 3 . These beliefs are the lottery generated by the ROL she submitted. After resolution in period 2, Lori's beliefs are $F_{2,3}=F_{3,3}$, which assign probability 1 to the consumption of the matched school.

In the general model, Lori's utility is the sum of two components: classical consumption utility, and news utility:

$$
\boldsymbol{u}_{\boldsymbol{t}}=m\left(\boldsymbol{c}_{\boldsymbol{t}}\right)+\sum_{\tau=t}^{T} N\left(F_{t, \tau} \mid F_{t-1, \tau}\right) .
$$

The term $m\left(\boldsymbol{c}_{\boldsymbol{t}}\right)$ corresponds to classical (reference-independent) consumption utility. Consumption utility is additively separable across dimensions, and the consumption-utility function in each dimension $k, m^{k}(\cdot)$, is assumed to be differentiable and strictly increasing. $N\left(F_{t, \tau} \mid F_{t-1, \tau}\right)$, defined below, is the "gain-loss" utility function. For $\tau=t$, it is Lori's "contemporaneous gain-loss" utility, which measures how she experiences consumption relative to what she believed entering period $t$; for $\tau>t, N\left(F_{t, \tau} \mid F_{t-1, \tau}\right)$ is her "prospective gain-loss" utility, which measures how Lori reacts to news about future consumption 8

The model defines $N(F \mid F)$ as follows. For any distribution $F$ over $\mathbb{R}$ and any $p \in(0,1)$ let $c_{F}(p)$ denote the consumption level at percentile $p . c_{F}(p)$ is essentially the inverse of $F(\cdot)$, and is defined by satisfying the following conditions:

$$
F\left(c_{F}(p)\right) \geq p
$$

\footnotetext{
8 Köszegi and Rabin (2009) have an additional parameter, $\gamma_{t, \tau}$, the weights Lori assigns to hearing news in period $t$ regarding her consumption in period $\tau \geq t$. In our analysis, we assume news about period-3 consumption (the only consumption period) to have the same weight in periods 1 and 2 , and therefore further normalize all $\gamma$ 's $=1$.
} 
Table 1: News Utility, Consumption Utility, and Beliefs in the Three Time Periods

\begin{tabular}{|c|c|c|c|c|c|}
\hline \multirow[b]{2}{*}{ Probability } & \multirow{2}{*}{$\begin{array}{c}t \\
\text { Learning } \\
1\end{array}$} & \multicolumn{2}{|c|}{$\begin{array}{c}t+1 \\
\text { Resolution }\end{array}$} & \multicolumn{2}{|c|}{$\begin{array}{c}t+2 \\
\text { Consumption }\end{array}$} \\
\hline & & $p$ & $1-p$ & $p$ & $1-p$ \\
\hline $\begin{array}{l}\text { Consumption in } \\
\text { Current Period }\end{array}$ & 0 & 0 & 0 & $x$ & 0 \\
\hline $\begin{array}{c}\text { Expectations } \\
\text { About } \\
\text { Consumption in } \\
t+2\end{array}$ & $\begin{array}{c}L= \\
\{x, p ; 0,1-p\}\end{array}$ & $\{x, 1\}$ & $\{0,1\}$ & $\{x, 1\}$ & $\{0,1\}$ \\
\hline News Utility & $\begin{array}{l}N^{k}(L \mid 0)= \\
\eta p m^{k}(x)\end{array}$ & $\begin{array}{c}N^{k}(x \mid L)= \\
\eta(1-p) m^{k}(x)\end{array}$ & $\begin{array}{l}N^{k}(0 \mid L)= \\
-\eta \lambda p m^{k}(x)\end{array}$ & $N^{k}(x \mid x)=0$ & $N^{k}(0 \mid 0)=0$ \\
\hline Consump. Utility & 0 & 0 & 0 & $m^{k}(x)$ & 0 \\
\hline
\end{tabular}

and

$$
F(c)<p \text { for all } c<c_{F}(p) \text {. }
$$

The gain-loss utility from changes in beliefs regarding the consumption in dimension $k$ is defined as

$$
N^{k}\left(F_{t, \tau}^{k} \mid F_{t-1, \tau}^{k}\right)=\int_{0}^{1} \mu\left(m^{k}\left(c_{F_{t, \tau}^{k}}(p)\right)-m^{k}\left(c_{F_{t-1, \tau}^{k}}(p)\right)\right) d p,
$$

where $\mu(\cdot)$ is a gain-loss utility function defined in the following way:

$$
\mu(x)= \begin{cases}\eta x & x \geq 0 \\ \eta \lambda x & x<0 .\end{cases}
$$

$\eta>0$ is the weight Lori assigns to changes in her beliefs, and $\lambda>1$ is the coefficient of loss aversion, which measures how losses are weighted relative to gains.

While the definition of $N(F \mid F)$ is notationally cumbersome, the intuition behind it is quite simple: Lori compares her old beliefs to her new beliefs percentile by percentile: in each period $t$, Lori compares the worst outcomes under $F_{t, \tau}^{k}(\cdot)$ to the worst outcomes under $F_{t-1, \tau}^{k}(\cdot)$, the second-worst outcomes under $F_{t, \tau}^{k}(\cdot)$ to the second-worst outcomes under $F_{t-1, \tau}^{k}(\cdot)$, and so on.

Table 1 summarizes Lori's utility and beliefs from a period- $t$ surprise lottery $L$ that will be resolved in period $t+1$. The lottery will give Lori consumption, in period $t+2$, of $x$ units in dimension $k$ with probability $p$ and 0 units otherwise. We normalize $m^{k}(0)=0$. 
Summing over the bottom two lines of the table, weighted by the corresponding probabilities in the top line, Lori's expected lifetime utility in dimension $k$ from this lottery is given by

$$
u^{k}=p(1+\eta) m^{k}(x)-p(1-p) \eta(\lambda-1) m^{k}(x)
$$

where the first term represents expected consumption utility and news utility (or gain-loss utility) from learning about the lottery, and the second term, which is always negative (because $\lambda>1$ ), represents expected news utility from the resolution of the lottery.

Although the model as written above has two parameters, equation (1) can be rearranged to have a single parameter, $\Lambda \equiv \frac{1+\eta \lambda}{1+\eta}$, sometimes referred to as the behavioral, or de facto, coefficient of loss aversion. However, it is common in the literature to discuss results in terms of $\lambda$ (rather than $\Lambda$ ) under the identifying assumption that $\eta=1$, and we follow this practice below. With this convention, equation (1) simplifies to

$$
u^{k}=2 p m^{k}(x)-p(1-p)(\lambda-1) m^{k}(x)
$$

\section{Predictions}

We start with a setting where Lori ranks $n$ different schools, and we analyze school flipping and omission. We postpone our analysis of funded positions to subsection 2.6.

\subsection{Defining the Problem}

Lori has preference over a set of schools $S=\left\{s_{1}, \ldots s_{n}\right\}$, ordered from most to least preferred. To get a match using DA, Lori submits her ranking over schools in an $l$-long list (ROL) with $0 \leq l \leq n$. Because she is uncertain about which school (if any) she will be matched with given a submitted ROL, choosing a ROL is, effectively, choosing a lottery over schools.

One important modeling assumption regards consumption dimensions; results depend on whether different schools belong to the same consumption dimension or to separate ones. Since, as mentioned above, we view a match with a program as potentially determining many aspects in Lori's life, we view different schools as different lifetime sequences, and model them as at least partially separate consumption dimensions $9^{9}$ This important assumption is simplifying and restrictive, equivalent to assuming that each school has one dominating

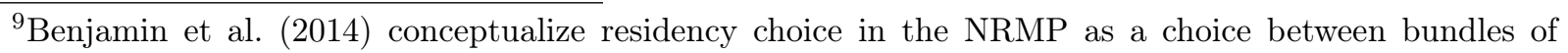
resedency features (such as prestige). They find that a participant's survey-elicited expectations regarding nine such features strongly predict her raking of residencies in her submitted ROL.
} 
dimension that in all other schools is at the same lower level.10

To analyze ROLs as lotteries, we distinguish between the probability of admission to a school conditional on proposing to it (that is, conditional on being rejected from all schools ranked above it), and the unconditional probability of admission to a school given a submitted ROL. The conditional probability distribution reflects the probabilistic beliefs held by an applicant regarding what the literature refers to as market conditions: her attractiveness at a specific school relative to unknown other relevant candidates, as well as the number of applicants the school desires to admit. Assume that applicant j's admission outcome at school $s_{i}$ is a deterministic function of a latent variable $y_{i j}$ reflecting $j$ 's attractiveness at $s_{i}$. Specifically, an applicant gets admitted (conditional on proposing) to school $s_{i}$ if $y_{i j}>y_{i}^{*}$ for some cutoff $y_{i}^{*}$. $y_{i j}$ takes the following form:

$$
y_{i j}=\beta^{\prime} x_{i j}+\epsilon_{i j}
$$

The variables $x_{i j}$ are applicant-school specific characteristics observed by the applicant, $\beta$ are (known) coefficients, and $\epsilon_{i j}$ is a random error term representing the applicant-school characteristics that are unknown to applicant $j$ (e.g., her recommendation letters' suitability for the school, the composition and idiosyncratic tastes of the admissions committee, and the relative strength of other candidates interested in $s_{i}$ ). Since we analyze the decision of a single DM, for ease of presentation we drop the subscript $j$. The probability of admission to a school conditional on proposing to it is therefore given by

$\operatorname{Pr}\left(\right.$ admission to $s_{i} \mid$ rejections from all schools ranked above $\left.s_{i}\right)=\operatorname{Pr}\left(\epsilon_{i} \geq y_{i}^{*}-\beta^{\prime} x_{i}\right) \equiv q_{i}$.

For simplicity, we assume independence of the idiosyncratic part of schools' preferences: $\epsilon_{i} \perp \epsilon_{l} \forall l \neq i$. This assumption would not hold if, e.g., candidates did not know how strong their letters of recommendation are in general. We effectively assume this possibility away: the only unknowns from our candidates' point of view are candidate-school-specific features that are independent across schools. This assumption simplifies the presentation and analysis, but we do not believe it meaningfully affects our conclusions 11

The set of possible ROLs, $\mathcal{R}$, contains all possible permutations of all sets contained in the power set of $S$. Given market conditions, submission of $r \in \mathcal{R}$ corresponds to a lottery

\footnotetext{
${ }^{10}$ Another de facto modeling assumption regards background risk (see Köszegi and Rabin, 2009, web appendix). Since a match determines a significant part of Lori's lifetime consumption, we view the match uncertainty as itself representing much of Lori's background risk; we effectively assume that additional background risk is negligible.

${ }^{11}$ Rees-Jones et al. 2019 induce in the lab the correlation that we assume away, between rejections from different schools. Incidentally, they find that participants fail to fully take it into account.
} 
over schools. We will later refer to the probability distribution that defines this lottery as the vector $\boldsymbol{p}(r)$. Under the assumptions we make on $\boldsymbol{q}=\left(q_{1}, \ldots, q_{n}\right), p_{i}(r)$ is the product of the probabilities of rejections from $s_{j}$ for all $j$ ranked above $i$, times the probability of admission to $s_{i}$. For example, if $r^{*}$ is the (consumption-utility) truthful list, $p_{i}\left(r^{*}\right)=q_{i} \cdot \prod_{j=1}^{i-1}\left(1-q_{j}\right) \cdot{ }^{12}$

We start by assuming $S=\left\{s_{1}, s_{2}\right\}$, so Lori's consumption vector is two-dimensional: $\left(s_{1}, s_{2}\right)$. (In subsection 2.5 we extend our main result to $n$ schools.) Denote $s_{i}=1$ if Lori is matched with school $s_{i}$. Note that $(1,1)$ is not possible, but $(0,0)$ is. Denote $m^{i}(1)=m_{i}>0$ the utility from being matched with school $s_{i}$, and normalize $m^{i}(0)=0$. Finally, WLOG assume $m_{1}>m_{2}$, i.e., Lori's consumption utility is higher for $s_{1}$.

The lottery that corresponds to each ROL $r$ is denoted by $L(r)$, and the ranking implied by $r$ is denoted by $\widehat{\succ}$. If Lori ranks only $s_{1}$, she gets the lottery

$$
L\left(s_{1}\right)=\left((1,0), q_{1} ;(0,0), 1-q_{1}\right)
$$

and similarly, if she ranks only $s_{2}$, her lottery is

$$
L\left(s_{2}\right)=\left((0,1), q_{2} ;(0,0), 1-q_{2}\right) .
$$

Finally, if she ranks truthfully, the corresponding lottery is

$$
L\left(s_{1} \widehat{\succ} s_{2}\right)=\left((1,0), q_{1} ;(0,1),\left(1-q_{1}\right) q_{2} ;(0,0),\left(1-q_{1}\right)\left(1-q_{2}\right)\right)
$$

with an analogous expression if she flips.

\subsection{Timing and Initial Beliefs}

As discussed in section 1, there are three relevant periods in this setting. In period 1, Lori learns that she can participate in the match by submitting a ROL; she plans which ROL to submit; and she submits (i.e., actually chooses) her planned ROL. By including learning, planning, and choosing/submitting in the same period (the only action period), we assume away the possibility that Lori plans a choice in one period, but implements her planned choice only in a later period. Our periodization therefore rules out, by assumption, the possibility of deviating from a planned choice, and hence the possibility of dynamic inconsistencies.

In the main text, we assume as above that Lori's inherited probabilistic beliefs entering period 1 regarding period-3 consumption are of zero consumption, and therefore her updated

\footnotetext{
${ }^{12}$ Other lists take a similar form, with a different order of indices. Whether Lori knows the correct probabilities associated with each list is of course irrelevant.
} 
period-1 beliefs, represented by the lottery implied by her submitted ROL, yield positive news utility. In appendix A, we allow Lori to hold different inherited beliefs entering period 1, and show that misrepresentation is still predicted in the cases we consider, although in some cases only under a narrower range of parameters. (We provide more detail in section 2.4 below.)

In period 2 uncertainty is resolved and Lori learns her match. Consumption occurs in period 3, which contains the rest of Lori's life. Using equation (2), Lori's utility from submitting a truthful ROL is therefore

$$
\begin{aligned}
U\left(L\left(s_{1} \widehat{\succ} s_{2}\right)\right) & =2 q_{1} m_{1}-q_{1}\left(1-q_{1}\right)(\lambda-1) m_{1} \\
& +2\left(1-q_{1}\right) q_{2} m_{2}-\left(1-q_{1}\right) q_{2}\left(1-\left(1-q_{1}\right) q_{2}\right)(\lambda-1) m_{2}
\end{aligned}
$$

and her utility from submitting a flipped ROL is a similar expression with the subscripts 1 and 2 flipped.

\subsection{Analysis of Flipping}

First, consider the case where Lori is required to include both schools on her list. Lori will choose to rank $s_{2} \widehat{\succ} s_{1}$ iff $U\left(L\left(s_{2} \widehat{\succ} s_{1}\right)\right)>U\left(L\left(s_{1} \widehat{\succ} s_{2}\right)\right)$, i.e., iff

$$
\frac{m_{1}}{m_{2}} \equiv m<\frac{3-\lambda+(\lambda-1) q_{2}\left(2-q_{1}\right)}{3-\lambda+(\lambda-1) q_{1}\left(2-q_{2}\right)}
$$

(and for all $m>1$ if only the denominator is negative).

For example, if $q_{2}=1$, $(3)$ reduces to $m<\frac{2+(\lambda-1)\left(1-q_{1}\right)}{2-(\lambda-1)\left(1-q_{1}\right)}$, which, with $\lambda=3$ and $q_{1}=\frac{1}{2}$, predicts that Lori will misrepresent her preference as long as $m<3$, that is, as long as the consumption utility from her preferred school is less than three times that from her lesspreferred school. Of course, with no loss aversion, DA is strategy-proof: with $\lambda=1$, the condition in (3) reduces to $m<1$, and, since $m>1$ by assumption, flipping never occurs.

Fixing $\lambda$, the RHS of (3) (which determines the upper bound of $m$ such that flipping is predicted) increases in $q_{2}$ and decreases in $q_{1}$. Note that given some $q_{2}$, as $q_{1} \rightarrow q_{2}$, the RHS of (3) approaches 1 (the no-flipping value). As $q_{1}$ decreases, the RHS of (3) increases, i.e., there is a wider range of values of $m$ where flipping occurs. Intuitively, a decrease in $q_{1}$ creates attachment to $s_{2}$ : expecting $s_{2}$ with high probability makes not matching with $s_{2}$ more costly, and Lori may choose to reduce this cost by increasing the probability of matching with $s_{2}$. This increase is achieved by flipping, which results in lower expected consumption utility but more certainty. As the value of $q_{1}$ approaches $q_{2}$, disappointment occurs with the same probability and the incentive to flip disappears, so Lori is predicted to 
Figure 1: Theoretical Predictions

$q_{2}=0.45$

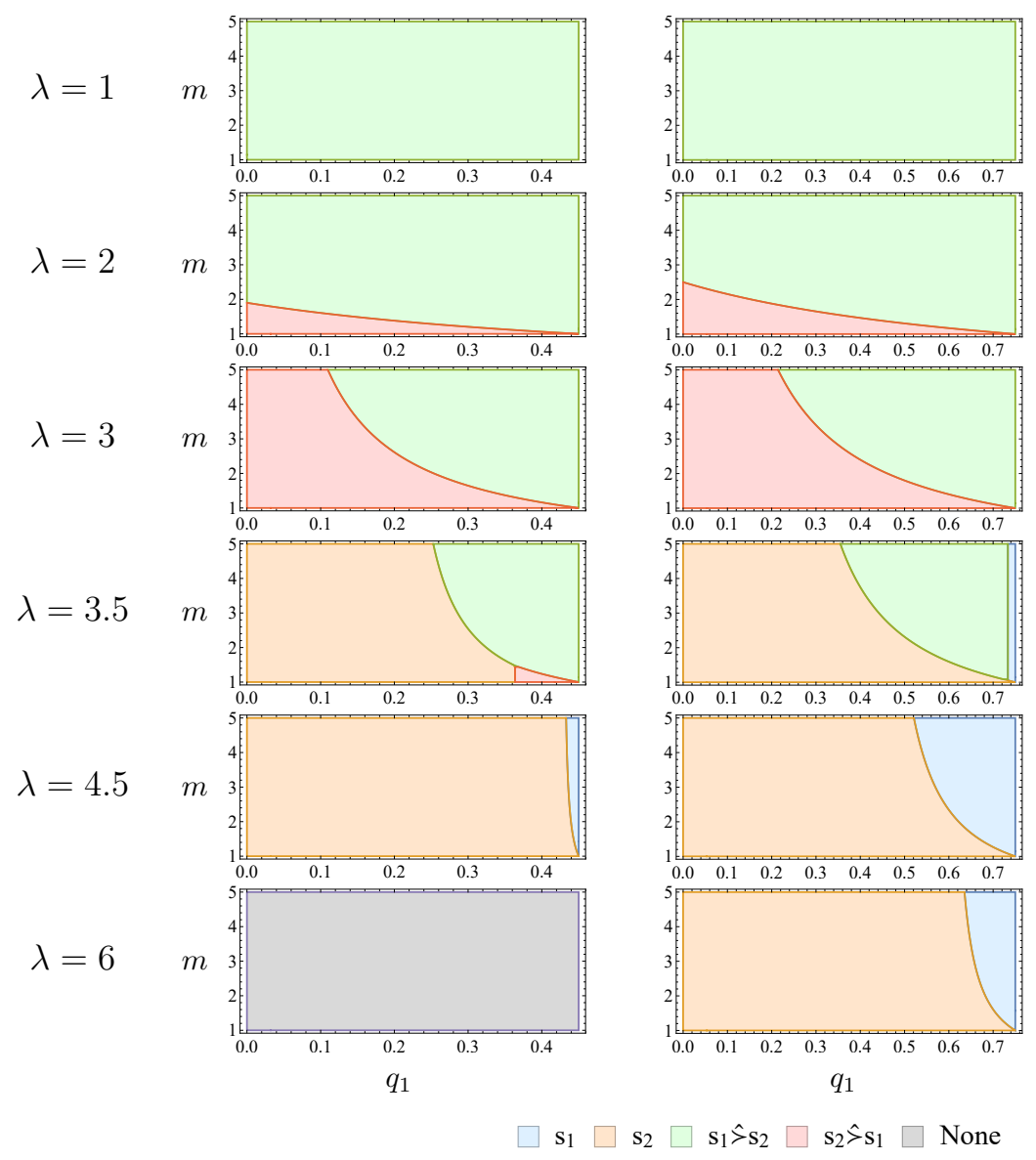

$q_{2}=0.95$
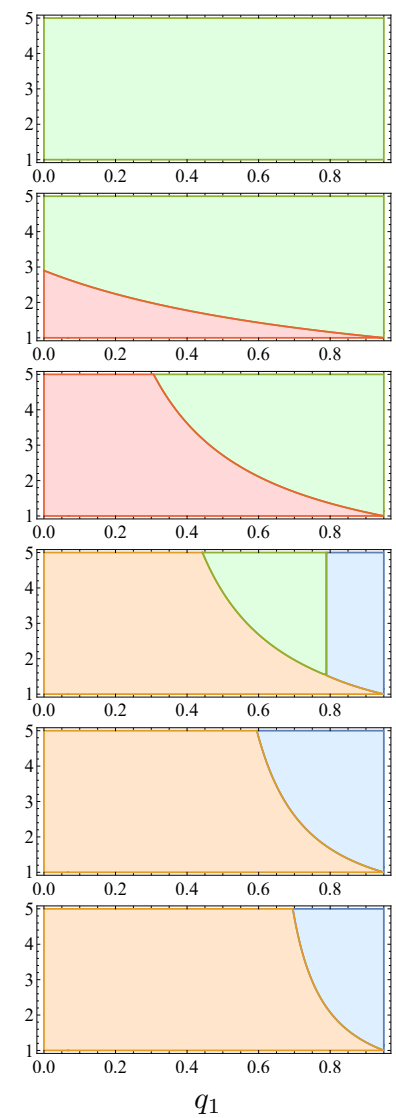

Notes: Model-predicted submitted list as a function of the model's parameters: each subplot shows the model's predictions for combinations of $q_{1}$ and $m$, fixing $q_{2}$ and $\lambda$. " $s_{1}$ " and " $s_{2}$ " denote lists containing only one school, with the other school omitted ( $s_{1}$ is the preferred school). "None" denotes an empty list.

rank schools by their consumption value 13

The top three rows of subfigures in figure 1 present the above analysis graphically. Each subfigure presents the range of combinations of $m$ and $q_{1}$ for which flipping is predicted for different values of $q_{2}$ and $\lambda$. As the top row shows, without loss aversion $(\lambda=1)$, no misrepresentation occurs. As $\lambda$ increases to 2 (second row) and then 3 (third row) and, for a given $\lambda$, as $q_{2}$ increases and as $q_{1}$ decreases, flipping is predicted for increasingly wider ranges of $m$.

\footnotetext{
${ }^{13}$ If we assume that $q_{1}$ decreases sufficiently faster than $q_{2}$, this analysis implies that, fixing preferences and loss aversion, flipping is more prevalent among those with weaker applications. For example, if $s_{1}$ is extremely selective relative to $s_{2}$ (because it is smaller or more prestigious), it is reasonable to assume that a flaw in the application (e.g. a somewhat weak letter of recommendation) hurts a candidate's chances of admission to $s_{1}$ much more than it hurts her chances of admission to $s_{2}$.
} 


\subsection{Analysis of Omissions}

We relax the complete-lists assumption and consider the case where Lori can choose to omit one (or both) of the schools. By plugging in the ROL-dependent probability, the utility $u^{k}$ from submission in each consumption dimension is given by (2) above. First, observe that for $\lambda \leq 3, u^{k}$ is always positive, implying that adding a school to Lori's ROL always increases her utility. Therefore, with $\lambda \leq 3$, Lori will always rank all available schools, and allowing for omissions does not change the analysis in 2.3 above. However, if $\lambda>3, u^{k}$ can be negative for a sufficiently small probability and a sufficiently high $\lambda$. As we discuss in 2.6 below, this violates FOSD. Note, however, that the sign of $u^{k}$ is independent of $m_{k}$.

We can analyze Lori's decision when allowing for omissions and assuming $\lambda>3$ in a few simple steps. First, we check if $U\left(L\left(s_{2}\right)\right) \geq 0$, or

$$
q_{2} \geq \frac{\lambda-3}{\lambda-1} \equiv \mathrm{p}
$$

One can think of the threshold $\mathrm{p}$, which will crop up repeatedly, as the severity of loss aversion $(\mathrm{b} \rightarrow-\infty$ as $\lambda \rightarrow 1 ; \mathrm{p} \rightarrow 1$ as $\lambda \rightarrow \infty)$. Since $q_{1}<q_{2}$, if this inequality does not hold, Lori will not submit a ROL at all: even though she wants to attend a school, she does not apply to any school in order to avoid disappointment. Second, we check if $U\left(L\left(s_{1}\right)\right)>0$, or $q_{1} \geq \mathrm{p}$. It is straightforward to see that if $q_{1}<\mathrm{b} \leq q_{2}$, Lori ranks only $s_{2}$. If both $q_{1}$ and $q_{2}$ are greater than $\mathrm{p}$, we check whether Lori will want to add $s_{1}$ to her list below $s_{2}$. Since $q_{1}\left(1-q_{2}\right)<q_{2}\left(1-q_{1}\right)$ by assumption, if $q_{1}\left(1-q_{2}\right)>\mathrm{p}$ holds, then Lori will list both schools, and the analysis continues as in 2.3 . If adding $s_{2}$ under $s_{1}$ is utility decreasing $\left(q_{2}\left(1-q_{1}\right)<\mathrm{p}\right)$, then Lori ranks only one school and compares between $s_{1}$ and $s_{2} .^{14}$ She submits $s_{2}$ if $U\left(L\left(s_{2}\right)\right)>U\left(L\left(s_{1}\right)\right)$, and $s_{1}$ otherwise. Note that this inequality depends both on $\lambda$ and the probabilities, which determine the degree of expected loss, and on $m$, which captures by how much Lori prefers $s_{1}$ over $s_{2}$. In words, Lori trades off her future expected loss and future consumption utility. Last, if $q_{2}\left(1-q_{1}\right)>\mathrm{p}$ but $q_{1}\left(1-q_{2}\right)<\mathrm{p}$ then Lori compares $s_{1} \widehat{\succ} s_{2}$ and $s_{2}$, which also trades off between future loss and future consumption.

The bottom three rows of figure 1 present the model's predictions for three values of $\lambda>3$. They show that comparisons between a full list and a partial list depend only on the probabilities (since the sign of $u^{k}$ is independent of $m_{k}$ ), while comparisons between full lists with $s_{1}$ versus $s_{2}$ on top depend both on the probabilities (which determine expected loss) and on $m$ (which captures consumption preferences).

\footnotetext{
${ }^{14}$ Note that the omission of $s_{2}$ is possible even when $s_{2}$ is a "safe school," that is, even when $q_{2}=1$. Since $q_{1}<1$, there is a chance that Lori will not get matched with any school, and yet, she chooses not to rank school $s_{2}$ at all.
} 
To summarize, with $\lambda \leq 3$, Lori is predicted to submit complete lists. She may show an endowment effect and flip the order of schools. This effect negatively depends on $m$. Intuitively, when the difference in utility from attending the two schools is small, flipping is less costly in terms of payoff, and is therefore more prevalent. Ranking two schools as opposed to one school adds another consumption dimension to utility, and therefore more potential disappointment. When $\lambda>3$, the effect of additional expected disappointment can dominate the effect of added expected consumption utility in this dimension. Lori may find it optimal to omit a school from her list, in order to avoid future disappointment from not getting it. This effect becomes stronger for larger values of $\lambda$ and smaller values of $q_{1}$ and $q_{2}$.

This entire analysis assumes that Lori enters the submission period with prior belief of 0. Appendix A shows the model's predictions assuming different prior beliefs. Specifically, we consider three possible sets of beliefs that Lori holds when entering period 1: (a) expecting to attend $s_{1}$ with probability 1 ; (b) expecting to attend $s_{2}$ with probability 1 ; and (c) expecting the lottery generated by ranking truthfully (that is, attending $s_{1}$ with probability $q_{1}$ and $s_{2}$ with probability $\left.\left(1-q_{1}\right) q_{2}\right)$. Relative to our zero-prior-expectations benchmark, if Lori expects, entering period 1 , to attend $s_{2}$, she is more likely to misrepresent (by flipping or by omitting $s_{1}$ ). If she expects to attend $s_{1}$, she is less likely to misrepresent, but misrepresentation is still predicted (especially for high values of $\lambda$ ), mostly by dropping $s_{1}$. Last, if Lori expects to rank truthfully, she is much less likely to misrepresent, but misrepresentation (by flipping) is still predicted for high $q_{2}$, as long as $q_{1}$ and $m$ are not too high.

\subsection{Misrepresentation with More Than Two Schools}

We prove that unless the conditional admission probabilities, $q_{1}, \ldots, q_{n}$, happen to weakly increase with preference, for a sufficiently small $m$, preference misrepresentation is strictly preferred.

Proposition. Let $S=\left\{s_{1}, s_{2}, \ldots, s_{n}\right\}$ be a set of schools, ordered from the most to least preferred, let $\boldsymbol{q}=\left(q_{1}, \ldots q_{n}\right)$ be the corresponding market conditions where $q_{j} \in(0,1) \forall n \geq$ $j \leq 1$, such that there exists at least one pair of schools $s_{i}, s_{i+1}$ such that $q_{i+1}>q_{i}$. If $\lambda>1$ then there exist $m_{i}, m_{i+1}$, such that the optimal ranking submission is non-truthful.

Proof. Denote the truthful ROL by $r^{*}$. Assume by contradiction that there exists such a pair $s_{i}, s_{i+1}$, and that for every $m_{i}, m_{i+1}, r^{*}$ is optimal. 
Denote $p_{j}\left(r^{*}\right)$ by $p_{j}$ :

$$
p_{j} \equiv\left(1-q_{1}\right) \cdot\left(1-q_{2}\right) \cdot \ldots \cdot\left(1-q_{j-1}\right) \cdot q_{j}=q_{j} \cdot \prod_{k=1}^{j-1}\left(1-q_{k}\right)
$$

Denote $\widetilde{q_{1}} \equiv p_{i}$ and $\widetilde{q_{2}} \equiv q_{i+1} \cdot \prod_{k=1}^{i-1}\left(1-q_{k}\right)$. Hence, $\widetilde{q_{1}}$ and $\widetilde{q_{2}}$ are the (unconditional) probabilities of getting matched with schools $s_{i}$ and $s_{i+1}$, respectively, if they are ranked at the $i$ th place (with the remainder of the list according to $r^{*}$ ). Notice that if we restrict our attention to dimensions $i$ and $i+1, \widetilde{q_{1}}$ and $\widetilde{q_{2}}$ are the same as $q_{1}$ and $q_{2}$ from the previous subsection. Since $q_{i+1}>q_{i}$ by assumption, we have $\widetilde{q_{2}}>\widetilde{q_{1}}$. We can now proceed with the analysis as in 2.3, focusing on schools $s_{i}$ and $s_{i+1}$ : If Lori flips $s_{i}$ and $s_{i+1}$ keeping the rest of her ranking unchanged, then $p_{j}$, and thus $u^{j}$, will remain constant for $j \neq i, i+1$. If $m_{i}, m_{i+1}$ satisfy (3), flipping $s_{i}$ and $s_{i+1}$ will strictly increase $u^{i}+u^{i+1}$, without changing the utility in all other dimensions, and therefore ranking truthfully is not optimal.

The intuition behind this result is simple: if preference ordering does not coincide with probability ordering, then we can always find a pair of schools that satisfy the conditions in 2.3, and with the appropriate $m$ find a ranking that strictly improves Lori's utility as compared to the truthful ranking ${ }^{15}$

\subsection{Funded Positions}

The misrepresentation analyzed above is difficult to identify in the field because the $m_{i}$ 's are not generally observable. We now analyze obvious misrepresentations - the omission or flipping of funded positions - which have been identified in the field.

We again assume two schools, only one of which, $s_{1}$, offers funding. We keep all assumptions and notation as above, and denote the funded position by $\widetilde{s_{1}}$. The probability of getting into the funded position conditional on getting into $s_{1}$ is $\pi$. The amount of funding is $\$ x$. Conditional on rejection from (the unfunded) $s_{1}$, the probability of admission to $\widetilde{s_{1}}$ (i.e., the probability of getting funding at $s_{1}$ ) is, naturally, $0{ }^{16}$ Note that there are now three different options to rank, which we assume to represent three different consumption

\footnotetext{
${ }^{15}$ As shown in appendix A if we consider different prior expectations, we may have to impose stronger assumptions on the market conditions. For example, if prior expectations are either $s_{1}$ for sure, or the lottery generated by ranking truthfully, then we have to assume that $q_{1}$ is sufficiently small and $q_{2}$ is sufficiently high.

${ }^{16}$ Formally (using the notation from 2.1), an applicant gets admitted with funding if $y_{i j}>y_{i}^{*}+c_{i}$, where $c_{i}>0$ is the difference between the cutoffs of the regular and the funded position. We denote $\pi \equiv \operatorname{Pr}\left(y_{i} \geq y_{i}^{*}+c_{i} \mid y_{i} \geq y_{i}^{*}\right)$. Rejection from the unfunded position implies $y_{i j}<y_{i}^{*}<y_{i}^{*}+c_{i}$.
} 
Figure 2: Upward and downward slopping utilities at $p=0$

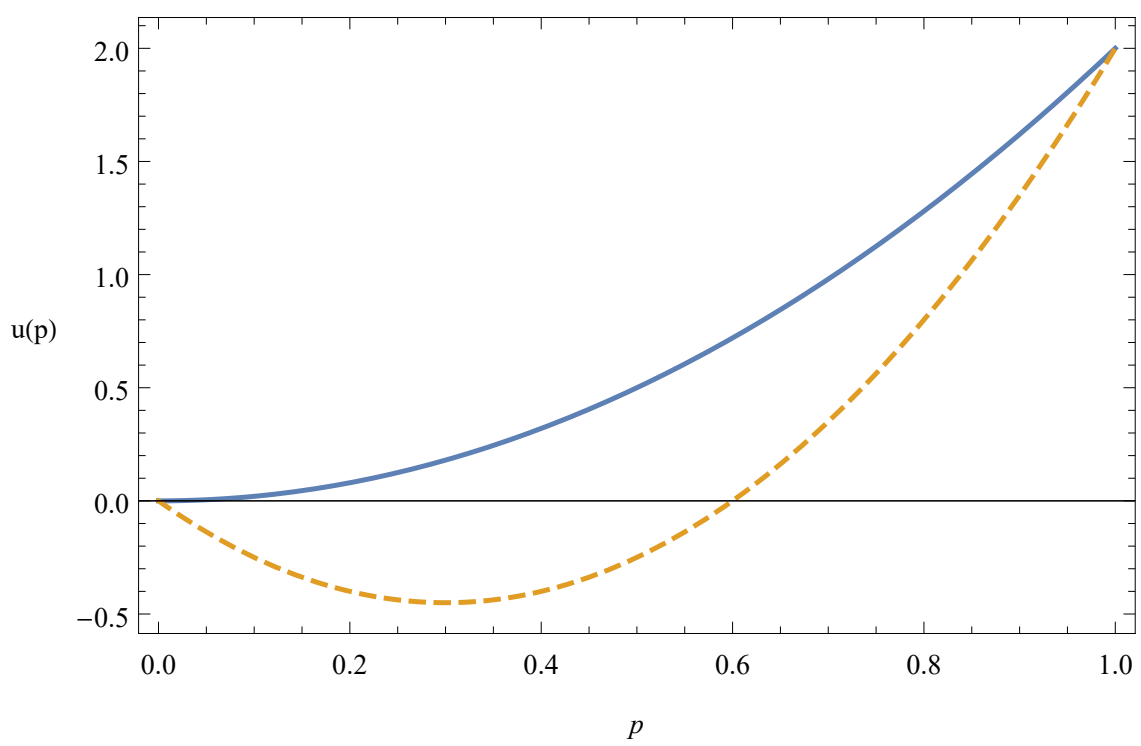

Note: Parameters: $m_{i}=1, \lambda=3$ (solid), $\lambda=6$ (dashed).

dimensions (one for each school and one for money). While this results in many more ranking options to analyze, we can show a simple condition for obvious misrepresentation. For simplicity, assume that the probabilities are such that $s_{1}$ is always ranked. Note that Lori is admitted to the funded program and gets a discount of $\$ x$ with maximum probability of $q_{1} \pi$. Plugging in these values into (2), her utility in the money dimension in this case is $u^{\text {money }}=2 q_{1} \pi m^{\text {money }}(x)-q_{1} \pi\left(1-q_{1} \pi\right)(\lambda-1) m^{\text {money }}(x)$, where $m^{\text {money }}(\cdot)$ is the consumption utility from money. As noted in 2.4. with $\lambda \leq 3$ this expression is always non-negative. However, if $q_{1} \pi<\mathrm{p}$ (which is possible only for $\lambda>3$ ), Lori never ranks $\widetilde{s_{1}}$ above $s_{1}$, i.e., she violates FOSD: she gives up on the possibility to win a prize with positive probability ${ }^{17}$

Figure 2 presents the RHS of (2) as a function of the probability in two possible cases (upward- and downward-sloping at $p=0$ ). It is easy to see that everything else equal, if $u^{\text {money }}$ is positive, a decrease in $q_{1} \pi$ decreases $u^{\text {money }}$, possibly changing its sign. As a result, ranking the funded position is suboptimal (if the expression is negative, it will remain negative with the decrease). Put differently, a decrease in $q_{1} \pi$ weakly increases the number of applicants who forgo the funding opportunity in order to avoid future disappointment.

As discussed in the introduction, this comparative static is consistent with an empirical

\footnotetext{
${ }^{17}$ In appendix $\mathrm{A}$ we allow for non-zero prior expectations entering the submission period. We show that the model predicts obvious misrepresentations only when Lori is sufficiently loss averse and sufficiently pessimistic (relative to $q_{1} \pi$ ), i.e., only if her $\lambda$ is high enough and her prior expectation to receive funding is low enough. On the other hand, if her priors are overoptimistic, the model predicts that Lori will never violate FOSD by omitting the funded position (independent of her $\lambda$ ). Interestingly, Rees-Jones and Skowronek (2018) find that all else equal, overconfident participants are more likely to submit truthful ROLs compared with non-overconfident participants.
} 
finding common to all relevant papers we know of: applicants with lower standardized test scores and therefore lower chances of being admitted to a funded position are more likely to obviously misrepresent (Hassidim et al., 2018; Shorrer and Sóvágó, 2018; Artemov et al., 2017). As shown by Shorrer and Sóvágó (2018), this correlation does not seem to be driven solely by misunderstanding or mistakes related to low ability. For example, they show that when the selectivity of some funded positions increased, more applicants flipped or omitted those positions. Interpreted as an exogenous shock to $\pi$, this suggests a causal relationship, as implied by our model ${ }^{18}$

If there are more than two schools, some of which offer funded positions with the same amount of funding $\$ x$, it is easy to see that at the optimum Lori's list will either rank each funded position above its non-funded counterpart, or none of the funded positions above its non-funded counterpart. This makes another empirical prediction that could be tested on a dataset that had pairs of funded and non-funded positions, where all funded positions offered the same amount of funding. We conjecture, but have yet to prove, that the results would be qualitatively similar when different schools offer different amounts of funding.

\section{Empirical Analysis}

In this section we reanalyze data on subjects' submitted ROLs from an experimental setting in which alternatives are allocated through a strategy-proof mechanism similar to DA. Of the experiments mentioned in the introduction, we chose to analyze Li (2017), a clean and carefully conducted recent experiment. Li's experiment has a simple setup (which minimizes potential subject misunderstanding and mistakes), and its data is publicly available. It is the only data we analyzed, which also limits the generalizability of our findings. As in other experiments, the alternatives that subjects rank are different sums of money, so that intrinsic preferences are obvious. Since all alternatives are on the same consumption dimension (money), the results from the previous section do not directly apply in this setup. However, we show below that the main prediction remains essentially unchanged: sufficiently loss-averse subjects $(\lambda>3)$ with lower chances of getting the highest prizes are predicted to rank them lower (violating FOSD), in order to reduce expected future disappointment.

\footnotetext{
${ }^{18} \mathrm{As}$ mentioned in the introduction, with this field evidence alone, we cannot rule out a theory in which applicants are more likely to make mistakes when those mistakes are less costly. In order to distinguish between such a theory and ours, we would need to lower $q_{1} \pi$ and increase $x$, keeping the expected utility from payoff, $q_{1} \pi m^{\text {money }}(x)$, constant; only our model would predict an increase in obvious misrepresentation. (Unlike this field evidence, the lab evidence we analyze in the next section does allow us to distinguish between the two theories.)
} 


\subsection{Experimental Design}

In Li's (2017) Strategy-Proof Random Serial Dictatorship (SP-RSD) treatment, four participants play ten rounds. In each round, four prizes are allocated between the participants. The prizes are drawn uniformly and without replacement from the set $\{\$ 0.00, \$ 0.25, \$ 0.50$, $\$ 0.75, \$ 1.00, \$ 1.25\}$.

At the start of each round, subjects observe the four prizes, and learn their priority score: an integer drawn uniformly and with replacement from 1 to 10. Subjects' priority scores are private information. After learning their priority scores, subjects have 90 seconds to rankorder each of the four prizes. They do this by typing a different number, from 1 (top) to 4 (bottom), next to each prize. Figure 3, reproduced from Li's (2017) instructions, shows the user interface. The mechanism is explained to participants in the instructions as follows 19

"After all the lists have been submitted, we will assign prizes using the following rule:

1. The player with the highest priority score will be assigned the top prize on his list.

2. The player with the second-highest priority score will be assigned the top prize on his list, among the prizes that remain.

3. The player with the third-highest priority score will be assigned the top prize on his list, among the prizes that remain.

4. The player with the lowest priority score will be assigned whatever prize remains.

If two players have the same priority score, we will break the tie randomly."

(We report the full instructions page from $\operatorname{Li}(2017)$ in appendix B) This allocation mechanism, serial dictatorship, is a restriction of DA to one-sided markets with priorities (that is, markets where only one side has heterogeneous preference rankings; the other side shares a universal priority ranking).

At the end of each round, subjects learn their realized priority - i.e., whether they are first, second, etc. - and the prize they receive. The instructions neither provide recommendations on how to play nor mention the existence of a dominant strategy. Eighteen groups of four players participated in this experimental condition, amounting to a total of 72 subjects and 720 subject-round observations. See $\operatorname{Li}(2017)$ for further details.

\footnotetext{
${ }^{19}$ Subjects have printed copies of the instructions, and the experimenter reads them aloud just before this part begins. Li (2017) reports that instructions are approximately at a fifth-grade reading level according to the Flesch-Kincaid readability test, a standard measure for how difficult a piece of text is to read.
} 
Figure 3: User Interface in Li (2017), SP-RSD Treatment

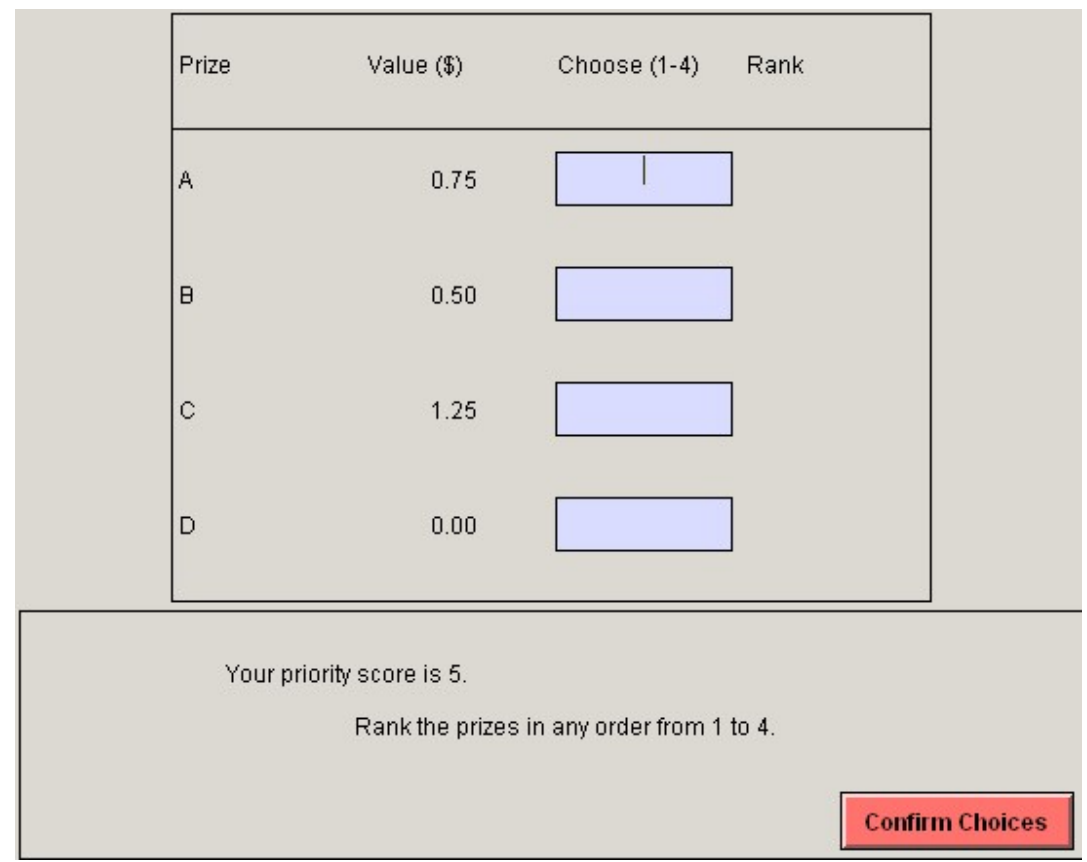

Note: Screenshot reproduced from Li (2017), web appendix.

\subsection{Theoretical Predictions}

In our model, the utility from a (unidimensional) lottery $\left\{x_{1}, \ldots, x_{4} ; p_{1}, \ldots, p_{4}\right\}$, with four monetary outcomes ordered from largest to smallest, is given by

$$
u(\boldsymbol{x}, \boldsymbol{p})=2 \cdot \sum_{i=1}^{4} p_{i} x_{i}-(\lambda-1) \sum_{i=1}^{4} p_{i} \sum_{j>i} p_{j}\left(x_{i}-x_{j}\right),
$$

under the following assumptions. First, utility is linear in money. Second, the timing within each round is: learning and submission in period 1 , resolution in period 2, and consumption in period 3. ${ }^{20}$ Third, we assume that subjects perceive prizes earned beyond the $\$ 20$ show-up fee as a positive surprise. In the multiround data, this assumption implies that subjects do not form new expectations about subsequent rounds; in the robustness analysis below we show that our empirical findings replicate separately for earlier and later rounds, as well as in a one-shot experiment.

The first term in (5) is the expected consumption utility plus period-1 news utility. It equals twice the lottery's expected value (EV). The second term is the expected period-2 news utility from the resolution of the lottery. It compares, in each contingency, the realized

\footnotetext{
${ }^{20}$ See the discussion on timing and initial beliefs in 2.2. We analyze the discrete-background-risk case as in Kőszegi and Rabin (2009).
} 
outcome $x_{i}$ against all other possible outcomes $x_{j}$, weighted by their probability. It equals $-(\lambda-1)$ times half the lottery's mean absolute difference (MD), a measure of statistical dispersion also known as the Gini mean distance (GMD). For a loss-averse player $(\lambda>1)$, this comparison term is negative (in expectation, the resolution of uncertainty yields negative news utility). Unlike in the multidimensional-lottery case analyzed in the previous section, in this unidimensional case where all outcomes are monetary amounts, there is no endowment effect: a subject who expects to receive a small amount but wins a larger one experiences only a gain - she does not also experience a loss from not getting the smaller amount. However, for a sufficiently high $\lambda$, the negative expected news utility from the resolution of the lottery may dominate the positive expected consumption utility, and a subject may thus prefer, ex ante, to give up a positive-expected-value lottery, violating FOSD.

To calculate her vector $\boldsymbol{p}$, a subject needs to combine two pieces of information. First, she needs to calculate the probability distribution of her realized priority within her group given her (privately known) priority score. For example, if her priority score is 10 (the highest), then the probability that she gets first priority is the probability that each of the three other players either got a score lower than 10, or got a 10 and lost the random tie breaker. Table 2 presents the probability distribution of realized priorities as a function of a subject's priority score ${ }^{21}$ While we do not believe that subjects actually calculate in their heads the exact probability in each cell of the table - a calculation complicated by tie breaking - we do believe that subjects have a reasonably good intuition regarding the approximate shape of the distribution. For example, with the highest priority score, it is very likely (86 percent chance) to place first, not very likely (13 percent) to place second, and extremely unlikely (1 percent) to place third of lower. With priority score of 5 or 6 , it is pretty likely (74 percent) to place second or third, and less likely (26 percent) to place first or fourth. And so on.

The second input into the vector $\boldsymbol{p}$ is a subject's beliefs regarding the ROLs submitted by the other subjects in her group. We start by assuming "face-value" beliefs: when a subject makes her ranking decision, she believes that the other subjects in her group always rank the prizes by face value. These beliefs are out of equilibrium because, as we show below, given these beliefs, the model predicts that some subjects will misrepresent (relative to face value). But they may be a natural point of departure for subjects, and an instructive benchmark that simplifies our presentation. We later replace this assumption by the assumption that subjects accurately predict the actual distribution of misrepresentation we observe. The two alternative assumptions yield qualitatively similar predictions.

We denote the truthful list by 1234, an abbreviation for 1st-2nd-3rd-4th. Under the beliefs implied by our face-value assumption - namely, that other subjects submit 1234 - a

\footnotetext{
${ }^{21}$ The probabilities were estimated through simulation using 1,000,000 draws.
} 
Table 2: Probability Distribution of Realized Priorities as a Function of Priority Score

\begin{tabular}{|c|c|c|c|c|}
\hline \multirow[t]{2}{*}{ Priority Score } & \multicolumn{4}{|c|}{ Probability of Realized Priority } \\
\hline & First & Second & Third & Fourth \\
\hline 10 & 0.86 & 0.13 & 0.01 & 0.00 \\
\hline 9 & 0.62 & 0.32 & 0.06 & 0.00 \\
\hline 8 & 0.42 & 0.42 & 0.14 & 0.02 \\
\hline 7 & 0.28 & 0.44 & 0.24 & 0.04 \\
\hline 6 & 0.17 & 0.41 & 0.33 & 0.09 \\
\hline 5 & 0.09 & 0.33 & 0.41 & 0.17 \\
\hline 4 & 0.04 & 0.24 & 0.44 & 0.28 \\
\hline 3 & 0.02 & 0.14 & 0.42 & 0.42 \\
\hline 2 & 0.00 & 0.06 & 0.32 & 0.62 \\
\hline 1 & 0.00 & 0.01 & 0.13 & 0.86 \\
\hline
\end{tabular}

Notes: Probability distribution of realized priorities in the experiment as a function of a subject's priority score. The probabilities were estimated through simulation using 1,000,000 draws.

subject who ranks a prize lower than its position by value, wins it with zero probability. For example, flipping the first two prizes, which we denote by 2134, means that if the subject is placed first or second, she wins the second-highest prize. If she is placed third (fourth), then the highest two (three) prizes are already taken by the time her list is processed, and hence she receives the third-highest (lowest) prize.

Subjects are required to rank all four prizes, so there are $4 !=24$ possible rankings. However, when a subject believes that all other players play 1234, these 24 rankings are reduced to 8 sets of rankings that yield 8 different lotteries. For example, if a subject ranks the lowest prize first, her lottery is degenerate: she wins the lowest prize with probability 1. Independent of her realized priority, the lowest prize will still be available by the time her list is processed. We denote by $4 \mathrm{XXX}$ the set of six lists that rank the lowest prize at the top. We use the same notation for the rest of the sets, with two exceptions. First, all lists with the second prize on top and the third prize ranked above the fourth yield the same lottery, regardless of the position of the first prize. We denote this set, $\{2134,2314,2341\}$, by $2 * 3 * 4 *$. Similarly, we denote by $2 * 4 * 3 *$ the set of lists with the second prize on top and the fourth prize ranked above the third, $\{2143,2413,2431\}$.

Figure 4a plots the utility from the eight ranking sets, conditional on a subject's priority score, for different values of $\lambda$ (assuming that prizes are equally distanced from each other). The utility in each column is normalized such that the value of each cell is in terms of withincolumn standard deviations from the mean. The cells marked with a black circle are the 
optimal (utility-maximizing) lists, given a priority score. For $\lambda=1$, the model is reduced to the standard model with linear utility from money. Naturally, in this case 1234 maximizes expected payoff for all priority scores, reflecting strategy-proofness. However, when the priority score is the lowest (1), all lists with 3 ranked higher than 4 give approximately the same expected payoff, because the probability of being placed first or second is extremely low, so all rankings that have 3 above 4 can be easily rationalized by the standard model with a small error term. In contrast, listing 4 above 3 is expected to be costly, because it makes the probability of winning the lowest prize very close to 1 instead of around 0.86 (with around 0.14 probability of winning a higher prize). The same pattern holds for $\lambda=2,3$.

With $\lambda>3$, however, the model predicts that subjects will intentionally misrepresent. Specifically, as the priority score goes down from 10 to 1, subjects will submit at the top of their ROLs values that are increasingly smaller than the highest prize. For example, a subject with $\lambda=4$ is predicted to rank truthfully when she gets a priority score 8-10, to flip the first two prizes when her score is 4-6, to rank the third-highest prize on top of her list when her score is $2-4$, and to rank the lowest prize on top when she gets the lowest priority score. For higher values of $\lambda$, this effect becomes weakly stronger, and subjects are predicted to rank lower amounts on the top of their list for even higher priority scores.

It is instructive to look at the extreme case of priority score $=1$. As noted above, a realized priority above third place is very unlikely in this case, less than 1 percent before rounding. If we approximate this probability to be exactly zero, and normalize the lowest prize to be zero, we get that the expected utility from ranking $4 \mathrm{XXX}$ is zero, and the expected utility from ranking 1234 is given by

$$
2 x_{3} p_{3}-x_{3} p_{3}\left(1-p_{3}\right)(\lambda-1)
$$

with $p_{3}$ the probability of raking third in terms of realized priority. When $p_{3}<\mathrm{p}$ ( $\mathrm{b}$ is defined in equation (4) on page 16), the second term dominates and the expression is negative. That is, the subject wants to completely eliminate the variance (and thus avoid the expected loss) and prefers 4 XXX, or any list with 4 above 3, over 1234. The same intuition can be applied to all other predicted violations of FOSD: subjects may wish to increase their chances of getting their first ranked choice, at the expense of their expected payoff. This effect becomes stronger for subjects with higher $\lambda$.

These predictions imply that, unless all players have sufficiently low $\lambda$, it is not a Nash equilibrium (NE) for everyone to play 1234: with the belief that all other players play 1234, players with $\lambda>3$ may find it optimal to rank lower prizes at the top of their lists. (Moreover, since such $\lambda>3$ players are the only ones without a dominant strategy, they 
Figure 4: Preferences over Lists and Empirical Distribution of Lists (Face-Value Beliefs) (a) Theoretical Predictions
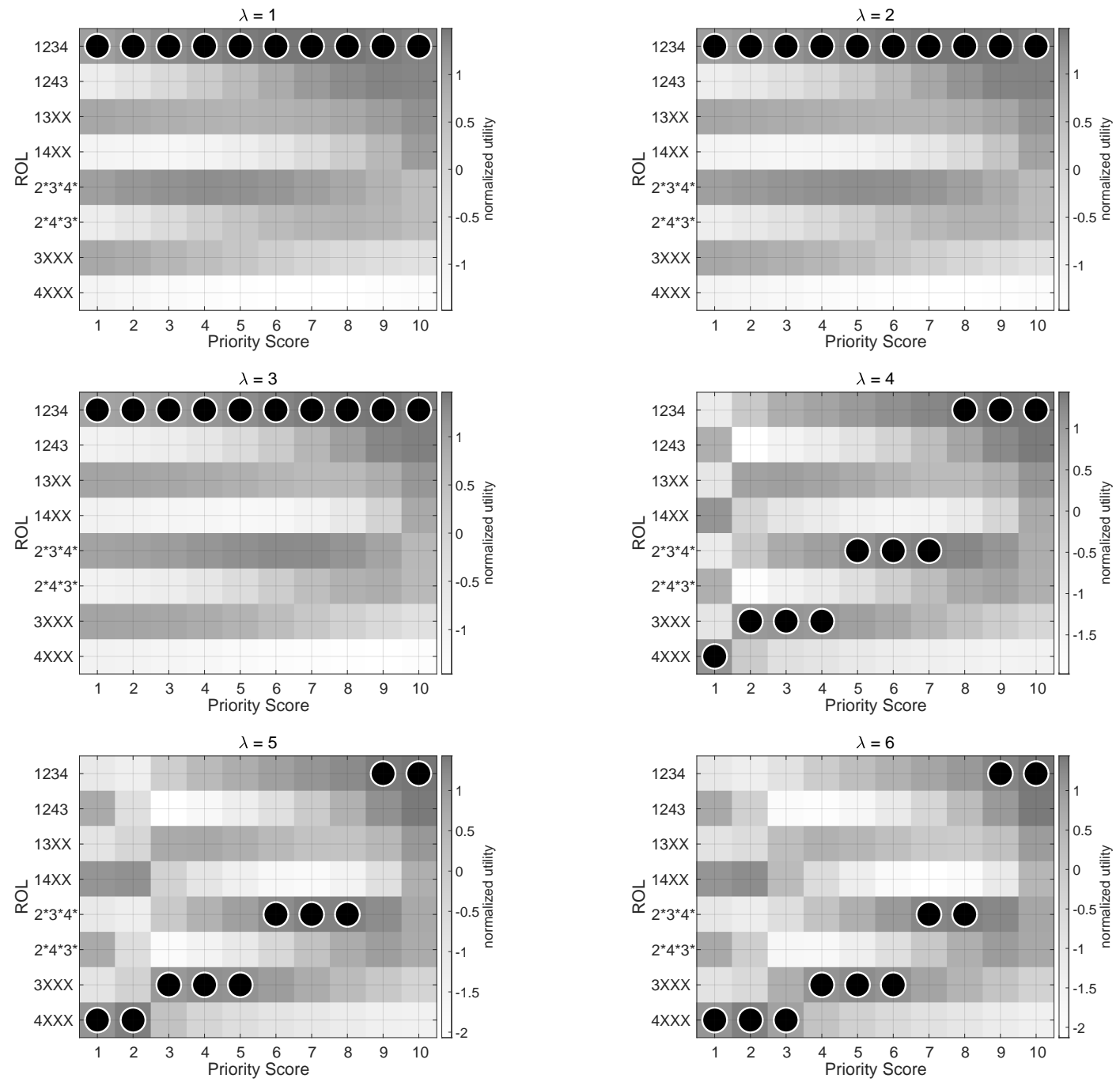

(b) Empirical Distribution (Multiround)
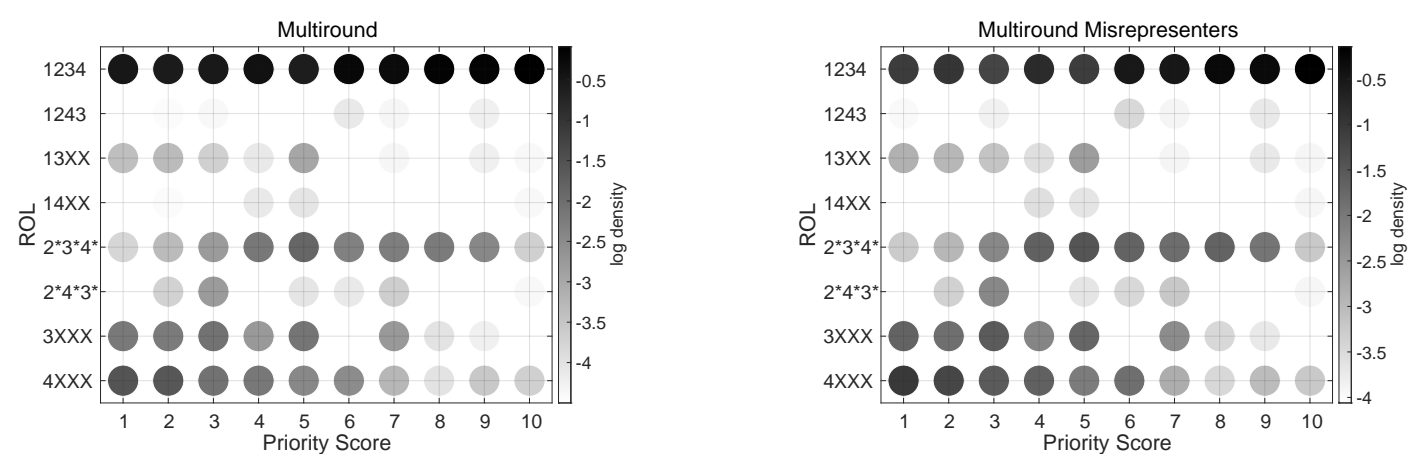

Notes: $13 X X=\{1324,1342\}, 14 X X=\{1423,1432\}, 2^{*} 3^{*} 4^{*}=\{2134,2314,2341\}, 2^{*} 4^{*} 3^{*}=\{2143,2413$, $2431\}, 3 X X X=\{3124,3142,3214,3241,3412,3421\}, 4 X X X=\{4123,4132,4213,4231,4312,4321\}$. Panel (a): Utility is normalized for each priority score. Distance between prizes is equal. Black circles: optimum. Panel (b): $N=720$ (Multiround), $N=440$ (Misrepresenters). Log density: share of observations per priority score. 
are the only ones whose beliefs about other players' behavior matter; it seems unlikely that these $\lambda>3$ players would believe that all players other than themselves have $\lambda \leq 3$.) We now analyze the model's predictions under the alternative assumption that subjects' beliefs are empirically correct: subjects can predict the empirical distribution of decisions for each priority score. When making their decision, subjects therefore take into account that other subjects (especially those who get a low priority score) may submit a list that is different from 1234. To the extent that the same empirical distribution of decisions that constitutes subjects' beliefs is also generated by these beliefs-i.e., the same distribution of decisions is also predicted by the model - the data could be consistent with a NE (for a certain distribution of $\lambda$ 's in the population).

For each score-list combination, we estimate the probability of winning each of the four prizes through simulation with the following data generating process (DGP). In each round, three priority scores (one for each of the three other players in the group) and four additional tie-breaking numbers are drawn uniformly. Conditional on the priority score drawn for each player, the probability of the player submitting each of the 24 possible lists is taken to be the same as its empirical density (across all player-rounds) for that priority score. We estimate the probability of winning each prize by simulating the outcome of each list-priority score combination 100,000 times. For example, we fix a priority score 5 and a list 1234 for one player, simulate the DGP described above, and use the proportion of rounds that player won the highest prize as the estimated probability of winning the highest prize when submitting 1234 with a priority score 5 . We repeat this process for all other score-list combinations to get a $4 \times 24 \times 10$ matrix that has the probability of winning each of the four prizes, for each 24 possible ROLs, for each of the 10 priority scores. This distribution is empirically correct in the sense that a player's belief about other players' behavior (and therefore about her chances of winning each of the prizes given her priority score and submitted list) is correct.

Figure 5 a presents the theoretical predictions under the assumption that subjects' beliefs are empirically correct. This assumption implies that each list results in a different lottery, because when other players may misrepresent, there is a positive probability of winning each of the prizes, for all score-list combinations. The figure therefore presents predictions for each of the 24 possible lists.

The predictions in this case are qualitatively similar to the predictions above: subjects with a high-enough $\lambda$ submit lists with lower prizes on top, the higher is their $\lambda$ and the lower is their priority score. However, under this assumption, for a given $\lambda$, the model predicts less misrepresentation; we therefore expand the range of $\lambda$ 's for which we make predictions. Intuitively, when other subjects submit non-1234 lists, the expected loss in payoff from misrepresentation is greater. In addition, while in the previous case ranking a 
Figure 5: Preferences over Lists and Empirical Distribution of Lists (Empirically Correct Beliefs)

(a) Theoretical Predictions
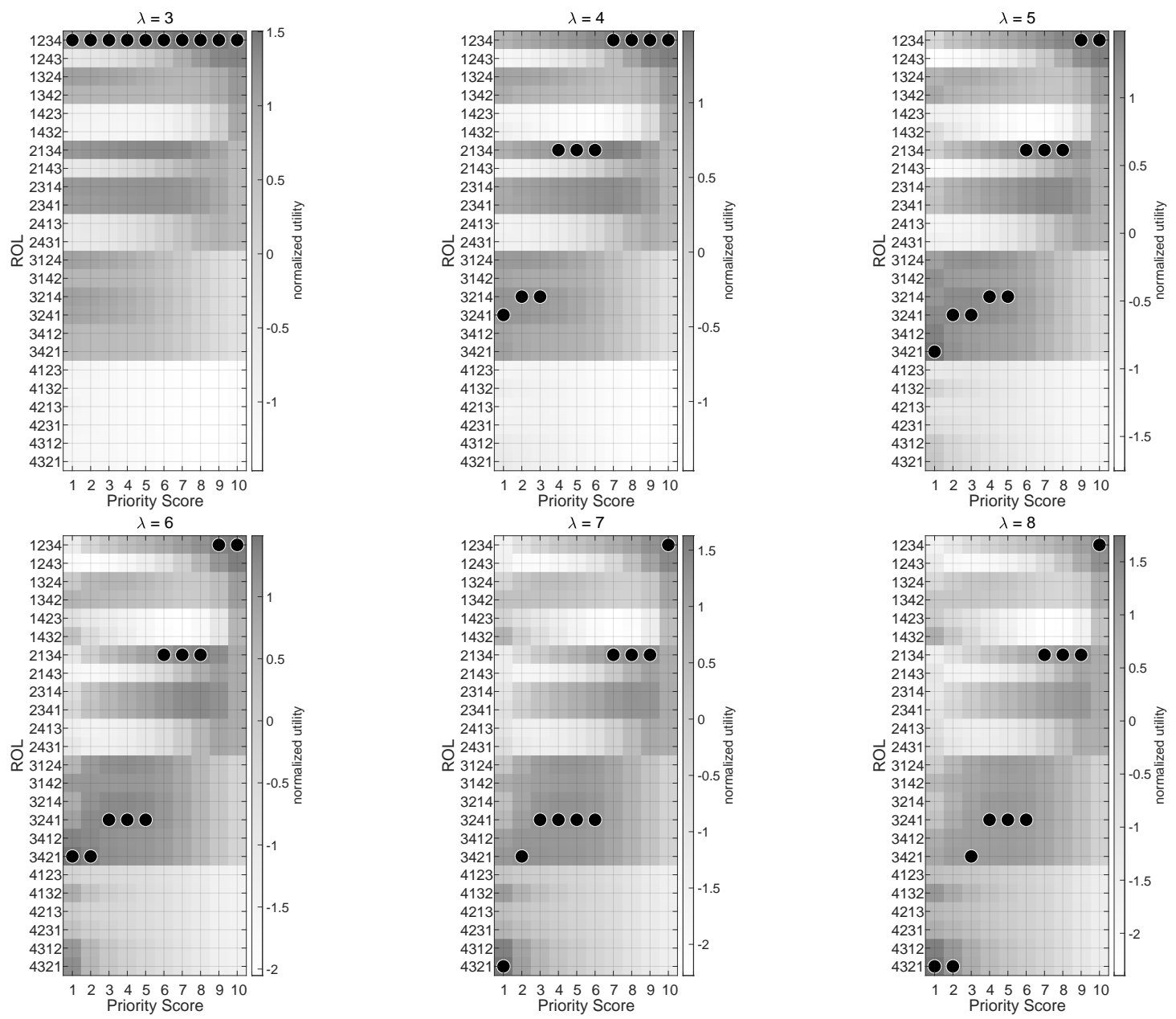

(b) Empirical Distribution (Multiround)
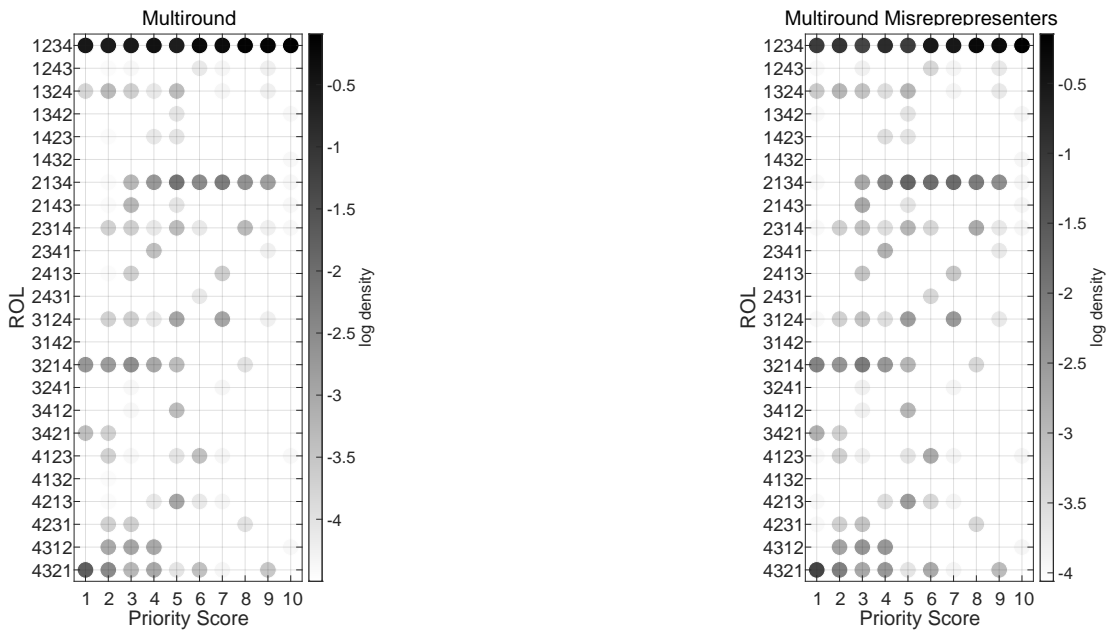

Notes: Panel (a): Utility is normalized for each priority score. Distance between prizes is equal. Panel (b): $N=720$ (Multiround), $N=440$ (Misrepresenters). Log density as a share of observations per priority score. 
prize lower than its relative value completely eliminates the probability of winning it, in this case the probability of winning the downranked prize remains always positive, so flipping is less effective in reducing the dispersion of the distribution and hence in avoiding loss. For this reason, complete reversal (4321) is predicted only for subjects with rather high $\lambda$ 's.

\subsection{Empirical Patterns}

In this descriptive subsection we analyze the empirical patterns and compare them with the theoretical predictions above. In the next subsection we move from eyeballing to econometric analysis: we formally test the fit of the model relative to the standard $(\lambda=1)$ model, and estimate the distribution of $\lambda$.

In Li's SP-RSD experiment, 36 percent of games do not end in the dominant-strategy outcome, meaning at least one player submitted an ex-post-payoff-relevant non-truthful list. At the subject-list level, in 29 percent of submitted ROLs, the four prizes are not listed in order of their value. Out of 72 subjects, 44 submitted a non-1234 list in at least one round. We later refer to this subsample as the misrepresenters (while continuing to refer to non-1234 lists as misrepresented submissions). The average loss from a misrepresented submission is $\$ 0.21$, around one-third of the average per-round earning (\$0.64). $\mathrm{Li}(2017)$ is focused on testing the performance of SP mechanisms against what he defines as Obviously Strategy-Proof (OSP) mechanisms, and is therefore mostly interested in the difference in the share of games that do not end in a dominant-strategy outcome. He offers a behavioral interpretation based on cognitively limited agents who fail to perform (a specific form of) contingent reasoning and therefore fail to recognize strategies as dominant. He does not focus on analyzing the data by either priority score or patterns of misrepresentation. 22

Hassidim et al. (2018) reanalyze Li's SP-RSD results and find that, in line with their finding from the field, priority score strongly predicts misrepresentation: a subject is more than 3 times as likely not to order the prizes by their values when she receives the lowest priority score relative to when she receives the highest score.

We can take the analysis one step further. The predictions above, summarized in figures $4 \mathrm{a}$ and 5a, predict players with specific levels of $\lambda$ and specific priority scores to submit specific (types of) lists. We now compare these predictions with the lists subjects actually

\footnotetext{
${ }^{22}$ In Li's other treatment, OSP-RSD, subjects sequentially choose, in order of their realized priority, a prize from among the remaining prizes - instead of submitting a list of preferences in advance. Only 7 percent of games in that treatment do not end in the dominant-strategy outcome, statistically significantly less than the shares of games that do in SP-RSD. In that treatment, our model has the same prediction as the classical, reference-independent-preferences model: a subject is predicted to pick the highest available prize when she is called to make the decision, regardless of $\lambda$. We note that OSP and EBRD are not theoretically equivalent in general; the mechanisms under which they yield similar predictions are yet to be characterized.
} 
Table 3: Empirical Distribution of Eight List Sets (Multiround)

\begin{tabular}{|c|c|c|c|c|c|c|c|c|c|c|c|}
\hline \multirow[t]{2}{*}{ ROLs } & \multirow[t]{2}{*}{ \#ROLs } & \multicolumn{10}{|c|}{ Priority Score } \\
\hline & & 1 & 2 & 3 & 4 & 5 & 6 & 7 & 8 & 9 & 10 \\
\hline 1234 & 1 & $61.1 \%$ & $57.1 \%$ & $58.8 \%$ & $67.7 \%$ & $55.2 \%$ & $79.0 \%$ & $74.4 \%$ & $85.7 \%$ & $84.3 \%$ & $91.3 \%$ \\
\hline 1243 & 1 & $1.1 \%$ & $1.2 \%$ & $1.3 \%$ & $0.0 \%$ & $0.0 \%$ & $1.6 \%$ & $1.3 \%$ & $0.0 \%$ & $1.4 \%$ & $0.0 \%$ \\
\hline $13 X X$ & 2 & $3.3 \%$ & $3.6 \%$ & $2.5 \%$ & $1.6 \%$ & $5.2 \%$ & $0.0 \%$ & $1.3 \%$ & $0.0 \%$ & $1.4 \%$ & $1.3 \%$ \\
\hline $14 X X$ & 2 & $0.0 \%$ & $1.2 \%$ & $0.0 \%$ & $1.6 \%$ & $1.7 \%$ & $0.0 \%$ & $0.0 \%$ & $0.0 \%$ & $0.0 \%$ & $1.3 \%$ \\
\hline $2 * 3 * 4 *$ & 3 & $2.2 \%$ & $3.6 \%$ & $6.3 \%$ & $11.3 \%$ & $15.5 \%$ & $9.7 \%$ & $10.3 \%$ & $10.7 \%$ & $8.6 \%$ & $2.5 \%$ \\
\hline $2 * 4 * 3 *$ & 3 & $0.0 \%$ & $2.4 \%$ & $6.3 \%$ & $0.0 \%$ & $1.7 \%$ & $1.6 \%$ & $2.6 \%$ & $0.0 \%$ & $0.0 \%$ & $1.3 \%$ \\
\hline $3 \mathrm{XXX}$ & 6 & $11.1 \%$ & $10.7 \%$ & $12.5 \%$ & $6.5 \%$ & $12.1 \%$ & $0.0 \%$ & $6.4 \%$ & $1.8 \%$ & $1.4 \%$ & $0.0 \%$ \\
\hline $4 \mathrm{XXX}$ & 6 & $21.1 \%$ & $20.2 \%$ & $12.5 \%$ & $11.3 \%$ & $8.6 \%$ & $8.1 \%$ & $3.8 \%$ & $1.8 \%$ & $2.9 \%$ & $2.5 \%$ \\
\hline Total & 24 & $100.0 \%$ & $100.0 \%$ & $100.0 \%$ & $100.0 \%$ & $100.0 \%$ & $100.0 \%$ & $100.0 \%$ & $100.0 \%$ & $100.0 \%$ & $100.0 \%$ \\
\hline$N$ & & 90 & 84 & 80 & 62 & 58 & 62 & 78 & 56 & 70 & 80 \\
\hline
\end{tabular}

Notes: Share of decisions as a percentage of choice situations with the same priority score. ROLs are grouped into sets as explained in figure $4 a$.

submitted. As above, for ease of presentation, we start by looking at the 8 sets of lists that result in the same lottery under the face-value-beliefs assumption, and compare their empirical distribution (conditional on priority score) to the theoretical predictions. Table 3 presents the empirical distribution; the numbers are also presented graphically in $4 \mathrm{~b}$. The full distribution of each of the 24 ROLs is reported in appendix C.

First, in line with the result reported by Hassidim et al. (2018), the density of 1234 lists drops sharply from 91 percent for subjects with the highest priority score to 61 percent for those with the lowest score. While not strictly monotonic - possibly due to the relatively small number of observations per score - the trend is quite clear. Indeed, Hassidim et al. (2018) show that on average, a 1-point decrease in priority score increases the probability of misrepresentation by 4 percentage points $(p$-value $<0.0001){ }^{23}$

Moving beyond Hassidim et al.'s (2018) analysis, the table further shows that other than 1234, the most common list sets are the three predicted by the model for $\lambda>3$ (see figures 4 a and 5a), namely $2 * 3 * 4 *, 3 X X X$, and 4 XXX. Specifically, consistent with the model with these higher $\lambda$ 's, $2^{*} 3^{*} 4^{*}$ is most common (9-16 percent) among subjects with a medium-tohigh (4-9) priority score, while 3XXX, and 4XXX are most common (7-21 percent) among those with a low-to-medium (1-5) score. Importantly, comparing figure $4 \mathrm{a}$ to $4 \mathrm{~b}$ and to table 3, the empirical distribution does not seem to be consistent with $\lambda=1$ subjects making random mistakes (independent of their cost) as can be seen from the clear patterns of misrepresentations. The empirical distribution also does not seem to be consistent with

\footnotetext{
${ }^{23}$ See their Table 5. Results are from a linear regression of a dummy for misrepresentation on priority score, with and without round and subject fixed effects, and with standard errors clustered at the subject level $(N=720)$.
} 
$\lambda=1$ subjects making mistakes with the probability of a mistake proportional to its cost. For example, at high priority scores (7-10), 1243 is the least costly mistake, and yet it is almost never submitted. In contrast, $4 \mathrm{XXX}$ is always the costliest mistake, and yet, for subjects with low priority scores (1-4) it is the second or third most common list. Similarly, $3 \mathrm{XXX}$ and $2^{*} 3^{*} 4^{*}$ are most common for priority scores at which they are not the cheapest mistakes for $\lambda=1$ subjects.

In figure 5b, we show for each priority score the empirical distribution of each of the 24 possible lists separately. Comparing $5 \mathrm{~b}$ to the predictions in 5a, the empirical patterns seem to be consistent with the theoretical predictions (for higher $\lambda$ 's) also with empirically correct beliefs. 2134 is most common among subjects with medium-to-high priority scores, while 3214 and 4321 are most common among low-to-medium-score subjects. The model sometimes predicts lists that are not empirically common (e.g., 3241), but the difference in utility between them and other, empirically common lists (e.g., 3214) is small and is therefore consistent with a small, unexplained error term. Similarly, the list 4312, which is never predicted by the model, seems to be somewhat common at low priority scores, but again, the difference in utility between this list and 4321 is negligible.

In summary, the observed submission patterns appear to be consistent with the model's predictions, which themselves appear qualitatively similar under either of our two alternative assumptions regarding subjects' beliefs about other subjects' play. Moreover, the empirical patterns seem to imply a substantial heterogeneity in $\lambda$, with a sizable fraction of subjects that are rather loss averse, i.e., have rather high $\lambda$ 's.

\subsection{Estimation}

We first present the results from a (multinomial) logit model with a single $\lambda$ for all subjects, and test our model against the standard $\lambda=1$ model. We then allow for heterogeneity in $\lambda$ by estimating the logit model for individual subjects separately, and by estimating a parametric distribution of $\lambda$ with a mixed-logit model.

\subsubsection{Empirical Specification}

Our empirical specification is based on equation (5) (on page 22). In its most general form, it assumes the following utility function for individual $i$ with a priority score $s$ who submits a list $l$ at round $r$ :

$$
U_{i s l r}=\alpha_{1} E V_{s l}+\alpha_{2 i} M D_{s l}+\varepsilon_{i l r}
$$

where $\alpha_{1}=2, E V_{s l}=$ the expected value of the (monetary) payoff from the lottery generated by a score $s$ and a list $l$ (corresponding to the first term in the RHS of (5)), $\alpha_{2 i}=\frac{1}{2}\left(1-\lambda_{i}\right)$, 
and $M D_{s l}=$ mean absolute difference, which equals twice the expected resolution gain-loss utility from this lottery (the second term in (5)). Since $U$ is defined only up to an affine transformation, setting $\alpha_{1}=2$ uniquely identifies $\lambda_{i}$. We keep the assumption that subjects' beliefs regarding other subjects' play coincide with the empirical distributions (conditional on score) of actual plays; results under the alternative, face-value-beliefs assumption are similar (see appendix D). $\varepsilon_{i l r}$ is an i.i.d. error term, distributed extreme value type I with 0 mean. We interpret $\varepsilon_{i l r}$ as subjects' mistakes; they are less likely as they become larger. ${ }^{24}$ Note that with $\lambda_{i}=1 \forall i$, we get $\alpha_{2 i}=0$, and the model reduces to the standard model where subjects maximize $E V$, but are allowed to make mistakes whose probability decreases as they become larger (and, therefore, costlier in utils).

With $\alpha_{2 i}=\alpha_{2} \forall i$, all subjects have the same coefficient of loss aversion $\lambda$, and the model is reduced to the standard logit model. In that case, the probability that a decision maker $i$ with a priority score $s$ chooses the list $l$ at round $r$ is

$$
P_{i s l r}=\operatorname{Prob}\left(\alpha_{1} E V_{s l}+\alpha_{2} M D_{s l}+\varepsilon_{i l r}>\alpha_{1} E V_{s k}+\alpha_{2} M D_{s k}+\varepsilon_{i k r} \forall k \neq l\right)
$$

and, under the assumptions made on $\varepsilon_{i l r}$, it simplifies to the usual expression

$$
P_{i s l r}(\alpha)=\frac{e^{\alpha^{\prime} x_{s l}}}{\sum_{k} e^{\alpha^{\prime} x_{s k}}},
$$

where we collectively refer to the parameters $\alpha_{1}$ and $\alpha_{2}$ as $\alpha$, and to the lottery attributes $E V$ and $M D$ as $x$. We estimate this model using Maximum Likelihood (ML).

\subsubsection{Logit Quantal Response Equilibrium}

Quantal response equilibrium (QRE; McKelvey and Palfrey, 1995) is an equilibrium notion with boundedly rational agents who might make errors when choosing which pure strategy to play, but whose probability of choosing any particular strategy is positively related to the payoff from that strategy. In a logit QRE (LQRE) specification, the strategies are chosen according to the logit probability (given in (7) with correct beliefs about other players' behavior ${ }^{25}$ Our estimated model can therefore be viewed as a LQRE with a non-standard utility function. We test its fit against a standard LQRE (with $\alpha_{2}=\frac{1}{2}(1-\lambda)=0$ ).

Table 4 column 1 reports our estimates: mean $\lambda=1.98(\mathrm{SE}=0.18)$, highly statistically

\footnotetext{
${ }^{24}$ As we explain below, this assumption, combined with empirically correct beliefs, make our model a Logit Quantal Response Equilibrium (LRQE) with a non-standard utility function.

${ }^{25}$ The standard LQRE model has an additional rationality parameter, which is identified only if all the parameters in the utility function are known.
} 
significantly different from 1, with a likelihood-ratio-test (LRT) statistic suggesting that the estimated model is more than $10^{7}$ times likelier than the $\lambda=1$ model $(p$-value $<0.0005)$.

Table 4: Mixed and Standard Logit Specifications

\begin{tabular}{|c|c|c|c|}
\hline & $\begin{array}{c}\text { (1) } \\
\text { Multiround } \\
\text { All } \\
\text { (Logit) }\end{array}$ & $\begin{array}{c}(2) \\
\text { One Shot } \\
\text { All } \\
\text { (Logit) } \\
\end{array}$ & $\begin{array}{c}(3) \\
\text { Multiround } \\
\text { Misrepresenters Only } \\
\text { (Mixed Logit, Normal Dist.) }\end{array}$ \\
\hline$\alpha_{1}($ normalized $)$ & $\begin{array}{c}2 \\
(0.09)\end{array}$ & $\begin{array}{c}2 \\
(0.18)\end{array}$ & $\begin{array}{c}2 \\
(0.13)\end{array}$ \\
\hline$\lambda$ & $\begin{array}{c}1.98 \\
(0.18)\end{array}$ & $\begin{array}{c}2.18 \\
(0.37)\end{array}$ & \\
\hline$\mu_{\lambda}$ & & & $\begin{array}{c}2.71 \\
(0.48)\end{array}$ \\
\hline$\sigma_{\lambda}$ & & & $\begin{array}{c}2.51 \\
(0.41)\end{array}$ \\
\hline Log-likelihood & -1657.81 & -453.95 & -1138.50 \\
\hline Likelihood ratio test & 32.24 & 11.47 & \\
\hline$p$-value & 0.000 & 0.001 & \\
\hline$N$ & 720 & 192 & 440 \\
\hline
\end{tabular}

Notes: The parameters were estimated through Maximum Likelihood (columns 1 and 2) and Maximum Simulated Likelihood with 1,000 draws (column 3). Stnadard errors in paranthesis. The probability of winning each prize is the simulated probability as described in 3.2 . Likelihood ratio test: $\mathrm{df}=1, H_{0}: \lambda=1$.

\subsection{Robustness and Heterogeneity}

\subsubsection{Simulation and Permutation Tests}

As discussed above, the empirical distribution of submitted ROLs (table 3 and figures $4 \mathrm{~b}$ and $5 \mathrm{~b}$ shows a distinctive pattern. When describing it in section 3.3 , we emphasized three independent features. First, misrepresentations account for a certain share (29 percent) of ROLs. Second, misrepresentations tend to concentrate at specific ROLs (e.g., 2134) rather than others (e.g., 3142). Third, misrepresentations tend to concentrate at lower priority scores. In this subsection we use three simulation/permutation tests, corresponding to three alternative data-generating models. The three maintain, respectively, only the first, the first 
and second, and the first and third above features of the data. We find that none of the three models could yield the LRT result above by chance (simulated exact $p$-value $<0.0001$ ), and conclude that our strong rejection of the $\lambda=1$ null in the previous subsection resulted from more than one of these three subsets of features.

In all three tests, we take the real dataset, and randomly assign a synthetic decision to each subject-score observation. In each test, the randomization of decisions follows a different rule that reflects an alternative data-generating behavioral model, as follows. First, we examine a naive trembling-hand model, in which subjects make randomly chosen errors, independent of their priority score. The probability of a tremble (i.e., of making an error) is taken to be the same as the empirical density of misrepresentations $(=209 / 720)$. To generate the data according to this model, we randomly choose 209 subject-score observations, and assign to them synthetic ROLs, chosen uniformly out of all 23 possible non-1234 ROLs; the rest of the observations are assigned the 1234 ROL. Second, we look at a more nuanced trembling-hand model in which subjects make the same errors they make in the real dataset. The probability of a specific tremble (e.g., 3142) is the same as its empirical density in the actual data, but, as in the previous model, trembles are independent of priority scores. To generate data according to this model, we randomly reshuffle the actually submitted ROLs across observations, breaking the pattern that relates specific priority scores to specific misrepresentations. Last, we consider a trembling-hand model in which subjects make random errors, and the probability of making a random error given a priority score is the same as the empirical density of misrepresentations at that priority score in the actual data. Datasets in this test contain the same number of (uniformly drawn) misrepresented lists per priority score as the real dataset 26

For each of the three alternative data-generating models, we synthesize 10,000 datasets, and apply to each dataset a LRT as in subsection 3.4.2 (we keep assuming that beliefs about others' play coincide with the empirical distribution of plays in the actual data). In each of the three tests, none of the 10,000 simulations yieds a LRT statistic higher than the actual-data LRT statistic (=32.24) from the previous subsection, implying a simulated exact $p$-value $<0.0001$ in each of the three tests.

\subsubsection{Earlier vs Later Rounds}

Subjects in multi-round experiments may make early mistakes that they learn to avoid with experience. In order to test whether our results are driven by subjects' behavior in

\footnotetext{
${ }^{26}$ This model may be interpreted as if ordering prizes by value entails some cognitive cost, and therefore at low priority scores (low expected payoff), subjects are more likely to submit a randomly (and uniformly) chosen ROL. If subjects are avoiding cognitive costs by, e.g., typing 1234 in order next to each prize (see the instructional screenshot in figure 3, it would be equivalent to drawing a ROL from a uniform distribution.
} 
early rounds, we analyze the first and last five rounds separately (appendix E). Figure E.1 replicates figures $4 \mathrm{~b}$ and $5 \mathrm{~b}$ for the two subsamples. While the share of misrepresentations drops slightly and insignificantly from 31 to 27 percent from the earlier to the later rounds, the figure shows that to the extent that any differences are visible, our theoretical predictions seem to fit the empirical patterns better in the later than in the earlier rounds. Applying the above logit model to the two subsamples (table E.1), we estimate $\lambda=1.86(\mathrm{SE}=0.28)$ and $\lambda=2.10(\mathrm{SE}=0.24)$, respectively, for the earlier- and later-rounds subsamples. In summary, subjects' inexperience in the earlier rounds does not drive our results.

\subsubsection{One-Shot Experimental Sessions}

After running the main experiment, Li (2017) ran additional experimental sessions comparing OSP- and SP-RSD. They consist of only a one-shot serial-dictatorship game, with stakes that are 12 times higher than in the main experiment: prizes are now drawn uniformly from $\{\$ 0, \$ 3, \$ 6, \$ 9, \$ 12, \$ 15\}$. 48 groups of 4 subjects participated in this one-shot SP-RSD experimental condition, generating a total of 192 observations. As a robustness check, we estimate our model on this additional dataset.

Li (2017) reports that in this treatment 40 percent of games do not end in the dominant strategy outcome - statistically identical $(p$-value $=0.80)$ to 44 percent in the first of the ten rounds in the original experiment. ${ }^{27} 33$ percent of the 192 subjects did not rank prizes by their values, and as a result misrepresenters lost an average of $\$ 2.30$, a little less than one-third of the average earning (\$7.30). In their re-analysis, Hassidim et al. (2018) show that, as in Li's original experiment, priority score strongly predicts misrepresentation also in this experiment.

Figure 6 shows the empirical distribution of ROLs, both grouped as in subsection 3.2 and list by list. Appendix Creplicates table 3 with this dataset. The results appear qualitatively similar to those from the main (ten-round) dataset. At the same time, with only around onefourth of the observations, many more cells are empty. Interestingly, subjects with the lowest priority score, who according to the theory should misrepresent most frequently, submit 1234 in 12 out of 13 cases (92 percent). While based on few observations, this finding appears to contrast with the ten-round results, where 1234 submissions account for only 55 out of 90 lowest-score cases (61 percent).

Table 4 column 2 presents the results from estimating, on the one-shot data, the same logit model as in column 1. Mean estimated $\lambda=2.18(\mathrm{SE}=0.37)$, and LRT $p$-value $<$

\footnotetext{
${ }^{27}$ In his one-shot OSP-RSD treatment, this number is 8 percent-again statistically identical ( $p$-value = 0.18 ) to 17 percent in the first round in the original experiment.
} 
Figure 6: Empirical Distribution of Lists (One Shot)

(a) Eight List Sets

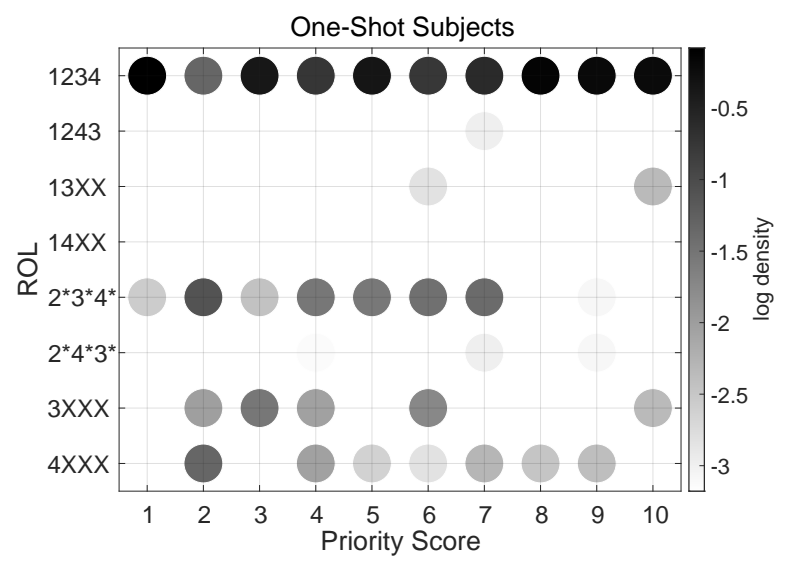

(b) All ROLs

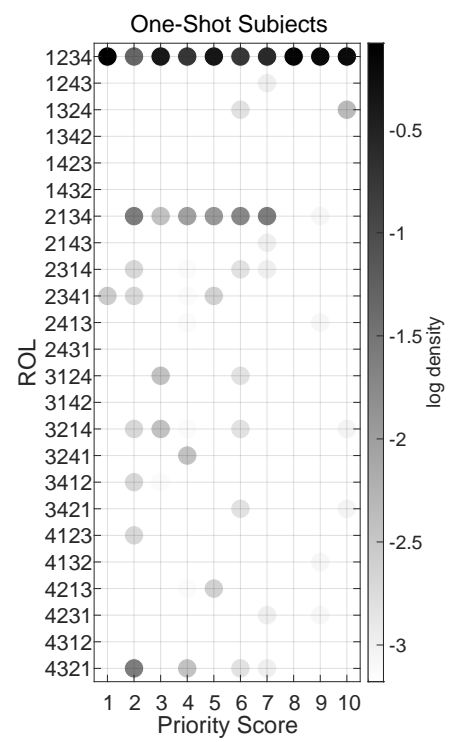

Notes: $N=192$. Log density as a share of observations with the same priority score. Panel (a): ROLs are grouped as explained in figure 4 a.

0.001 (against the $\lambda=1$ null). ${ }^{28}$ Overall, the results from this dataset are similar to those in column 1.

\subsubsection{Heterogeneity}

As discussed above, the empirical submission patterns summarized in figures $4 \mathrm{~b}$ and $5 \mathrm{~b}$ are consistent with substantial heterogeneity in $\lambda$. To investigate this heterogeneity, we start by estimating (6) at the individual level. Specifically, we use the 10 decisions made by each of the 44 misrepresenters to estimate $44 \lambda_{i}$ 's. For the remaining 28 subjects who submit 1234 in all 10 rounds, $\lambda_{i}$ is not identified. ${ }^{29}$ Towards the end of this subsection, we report full-sample statistics under different assumptions about the 28 unidentified $\lambda_{i}$ 's.

Figure 7 shows, first, a dashed-outline histogram of the 44 estimated values of $\lambda_{i}$ (mean $=$ $2.98, \mathrm{SD}=4.63$, median $=3.40$, and 84 percent of the 44 estimates $>1)$. It shows substantial

\footnotetext{
${ }^{28}$ For probability beliefs, these estimates use the same simulation results as in column 1, namely those based on the main dataset (with 720 choices). However, since the distribution of choices in the one-shot dataset (with 192 choices) is rather similar, estimating beliefs from that dataset instead yields essentially the same estimates (mean $\lambda=2.26, \mathrm{SE}=0.37$ ). Similarly, as reported in appendix $\mathrm{D}$, estimating the model under the face-value-beliefs assumption yields a one-shot point estimate that is very close to the main-dataset one, albeit with larger SEs and no statistically significant LRT result.

${ }^{29}$ It is easy to show that for these subjects, the likelihood function is unbounded.
} 
heterogeneity, with estimates ranging from - 20.86 to 12.5330 However, the 44 estimates vary widely in how precise they are, with standard errors ranging from 1.02 to 45.46. A second, solid-outline histogram therefore weights each point estimate by the inverse of its standard error. The weighted histogram effectively eliminates the most extreme outliers, is more concentrated, and is slightly shifted to the left (mean $=2.88, \mathrm{SD}=2.43$, median $=3.01$, and 80 percent of the weighted distribution $>1$ ). An additional solid curve plots a kernel estimate of the weighted distribution.

Figure 7: Distribution of $\lambda$ (Misrepresenters Only)

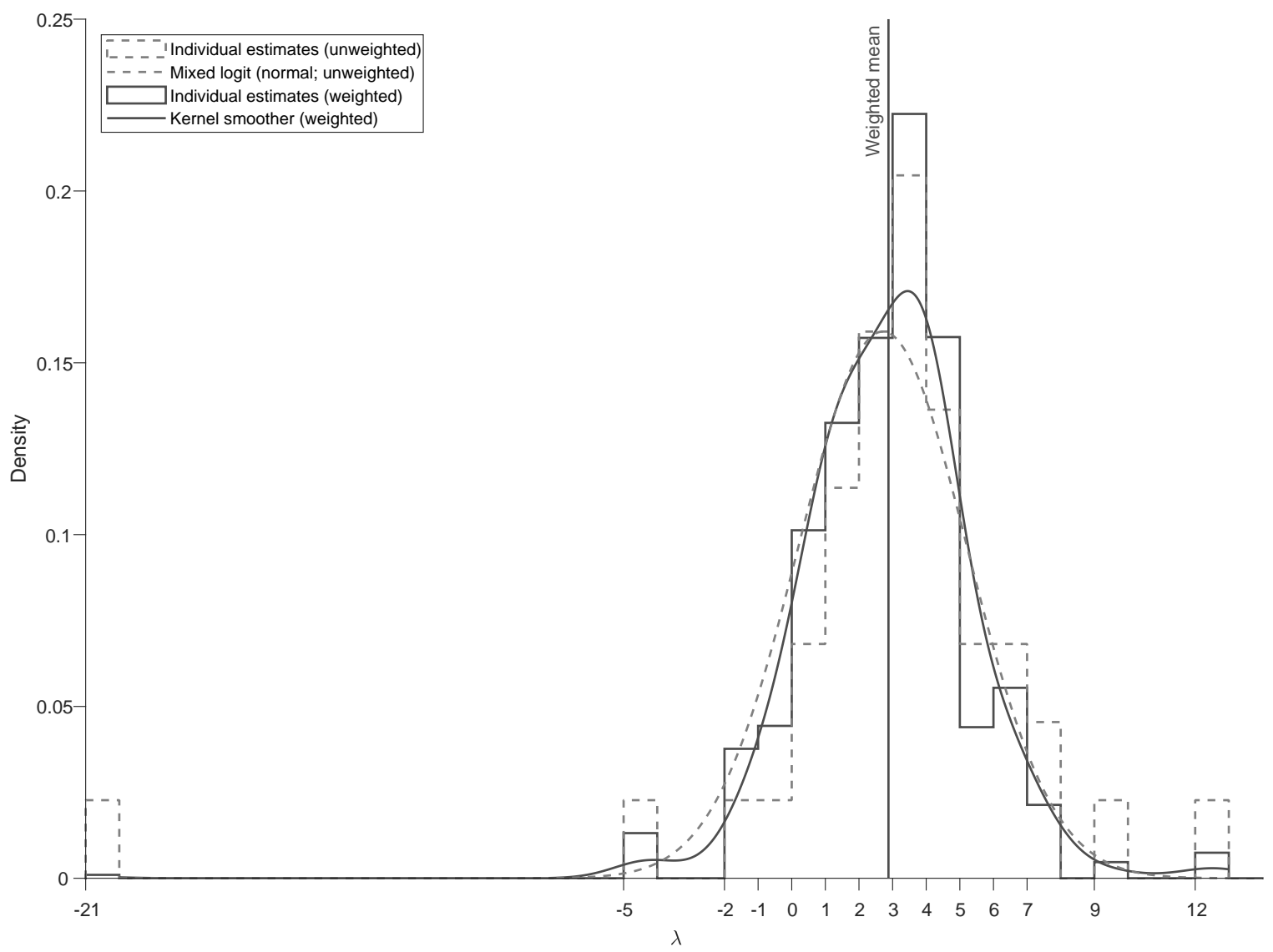

Notes: Unweighted (dashed outline) and weighted (solid outline) histograms: individual-level estimates of $\lambda_{i}$ for the 44 misrepresenters. Each observation is a point-estimate obtained by fitting equation (6) for each subject in the misrepresenters subsample, using her 10 decisions. Weights are the inverse of the SE of each point estimate. Solid curve: kernel fit of the weighted distribution (bandwidth $=1.02$ ). Dashed curve: mixed-logit estimate with normal mixing distribution (unweighted).

\footnotetext{
${ }^{30}$ Two of the 44 subjects make choices that imply aversion to money: they rank the lowest prize first in their highest-priority-score rounds. Their individual logit estimates therefore yield negative $\alpha_{1}$, the coefficient on EV. While such behavior cannot be explained by any model we are aware of, for completeness we treat these estimates like all others: we mechanically normalize their $\alpha_{1}=2$ and estimate their $\lambda_{i}$ 's $(=1.99$ and 7.45$)$. Dropping them from the sample affects our results in this section only trivially (for example, the mean above changes to 2.90 , and the median remains unchanged).
} 
Finally, eliminating all but two degrees of freedom, the figure shows a dashed-line normal distribution estimated on the raw data - the 440 choices of the 44 misrepresenters - using a mixed-logit (or random-coefficients) specification. Going back to (6), we specify a density of the distribution of $\alpha$ in the population of misrepresenters - a mixing distribution - denoted $f(\alpha \mid \theta)$, where $\theta$ denotes distribution parameters. The mixed-logit probability now takes the following form:

$$
P_{i s l r}(\theta)=\int\left(P_{i s l r}(\alpha)\right) f(\alpha \mid \theta) d \alpha=\int\left(\frac{e^{\alpha^{\prime} x_{s l}}}{\sum_{k} e^{\alpha^{\prime} x_{s k}}}\right) f(\alpha \mid \theta) d \alpha,
$$

where the second equality follows from equation (7). After specifying the functional form of the mixing distribution $f(\cdot)$, we estimate the parameters $\theta$ using simulation. The Maximum Simulated Likelihood Estimator (MSLE) is the value of $\theta$ that maximizes the simulated likelihood function. As in the standard logit model in equation (6), we normalize the fixed coefficient $\alpha_{1}$ to 2. We estimate (6) with MSL following the simulation and estimation methods described in Train (2009), using 1,000 draws for the simulated probabilities. Because the bell-shaped curve of the kernel estimate in figure 7 resembles a normal distribution, we choose a normal specification for $f(\cdot)$.

The (unweighted, two-parameter) normal distribution estimated with the mixed-logit model lies essentially on top of the (weighted, nonparametric) kernel distribution, and its implied summary statistics are similar to those of the weighted distribution of $\lambda$ 's. Table 4 column 3 reports our estimates: mean $=2.71(\mathrm{SE}=0.48), \mathrm{SD}=2.51(\mathrm{SE}=0.41)$. The high statistical significance of the SD of $\lambda$ implies that the null of no heterogeneity in $\lambda$ is easily rejected - even among this (potentially more homogenous) subsample of 44 misrepresenters. These mixed-logit estimates imply that 75 percent of misrepresenters have $\lambda>1$.

We can now translate these misrepresenters' statistics to full-sample statistics, under different assumptions regarding the 28 subjects who never misrepresent (and whose individual $\lambda_{i}$ 's are therefore not identified). We focus on means and medians, and consider three natural benchmark assumptions: the 28 unidentified $\lambda_{i}$ 's $=0$ (the lower bound to loss aversion without loss loving), 1 (loss neutrality, i.e., no reference dependence), and 3 (the upper bound to loss aversion without FOSD violations). For the unweighted distribution, under these three assumptions, mean $\lambda$ for the full 72-subjects population $=1.82,2.21$, and 2.99 , respectively; median $=1.29$ (assuming unidentified $\lambda_{i}{ }^{\prime} \mathrm{s} \leq 1$ ) and 3. For the weighted distribution, assuming a mass of $28 / 72$ at 0,1 , and 3 , weighted mean $=1.76,2.15$, and 2.93; weighted median $=0.98,1$, and 3 , respectively. For the estimated normal distribution of misrepresenters, assuming the same mass of $28 / 72$ at 0,1 , and 3 , mean $=1.65,2.04,2.82$, 
and median $=0.43,1$, and 3 , respectively.

This range of mean estimates of $\lambda$, from 1.65 to 2.99 , is similar to the range of past estimates in comparable subject populations, albeit using different versions of the model (see, e.g., Gill and Prowse, 2012; Sprenger, 2015 and, more recently, Goette et al., 2018, Chapman et al., 2018). However, such comparisons should be interpreted with caution. These past estimates are based on data from different settings with different strengths of the expectations manipulation. They also use different estimation methods.

\subsection{Discussion}

Throughout this section, we interpret the evidence of FOSD violations as at least partly intentional. Could this evidence instead result from experimental subjects' misunderstanding of the setting, or inattention to its implications regarding optimal behavior?

We argued above that subjects are unlikely to misunderstand Li's repeated RSD experiment. Still, subjects may be used to mechanisms that resemble the Boston mechanism ("first come first served"), and may inattentively follow common rules of thumb. They may therefore intuitively behave as they optimally would in a Boston version of RSD even if they do not misunderstand the setting. This possibility may explain why subjects aim for the prize they believe they are most likely to win, as we observe in the data. But this possibility is not supported by other features of the data. First, if subjects understand merely that each of the four players is allocated one of the four prizes, aiming for the worst possible outcome-i.e., choosing any ROL from the set $4 \mathrm{XXX}$ - is a weakly dominated choice under any mechanism that uses a monotone allocation rule. Yet ROLs from this set account for 10 percent of the 720 subject-round observations, and 34 percent of the 209 misrepresentations. Second, even if subjects do not understand that each player gets a prize, and fear that unless they aim for the smallest prize they may end up with no prize at all, submitting 4XXX is still weakly dominated when the smallest prize is $\$ 0$. Yet in 520 observations the smallest prize is $\$ 0$, and in 11 percent of them (35 percent of misrepresentations in this subsample), subjects submit 4XXX. In other words, a third of the misrepresentations cannot be explained by Boston-like intuitions or rules of thumb.

But if the FOSD violations analyzed in this section are driven by expectations-based loss aversion rather than by mistakes or inattention of this type, should we not expect to find similar violations in other experiments?

The rejection of a small chance of winning a prize appears opposite to a central feature of Prospect Theory that the EBRD model leaves out: the idea of probability weighting. With probability weighting, a small chance is subjectively overweighted as a larger chance, and 
therefore should be chosen more than implied by its actual expected value, rather than less. What might explain why so many of the subjects have (in our interpretation) shown a dislike of small chances of gains, so much as to violate dominance? The explanation that we believe is most important is simple: people are different. As discussed in the previous subsection on heterogeneity, under arguably plausible assumptions, our findings suggest that only a minority of Li's subjects have the high estimated $\lambda$ 's required for exhibiting FOSD violations. More generally, the estimates we report are fully consistent with a majority of Li's subjects finding small-chance gains appealing. Similarly, it is possible that in experiments that focus on investigating sample averages of probability-weighting parameters, the distribution of $\lambda$ is similar to what we estimate in Li's data.

Heterogeneity of experimental settings could also play a role, and could also explain a wide range of findings in the probability-weighting literature itself (Fehr-Duda and Epper, 2012; Barberis, 2013). However, the settings under which each of these apparently opposite behaviors dominates are yet to be fully understood (and modeled). At present, we can only speculate that there might be something about some settings that causes subjects to focus on, and overweight, the chance of a positive surprise, while in other settings something causes (the same or other) subjects to focus on other things, including the prospect of a potential disappointment, to the extreme of exhibiting FOSD violations.

While most economics experiments do not look for - and frequently use designs that do not allow for-FOSD violations, evidence of FOSD violations that seem to reflect intentions rather than slips has been found in past experiments. Most notably, Gneezy et al. (2006) find evidence of what they call the uncertainty effect: a lottery over different non-monetary prizes or deferred payments is valued less than the certainty of the lottery's worst outcome. ${ }^{31}$ While the original finding has been challenged (Rydval et al., 2009, Keren and Willemsen, 2009) and may be sensitive to small implementation details, it is replicated by Simonsohn (2009), who also argues that it is unlikely to be caused by misunderstanding or confusion.

Most evidence for the uncertainty effect relies exclusively on between-subjects design, in which the subjects who evaluate the certain outcomes are not those who evaluate the lottery that mixes them-as opposed to our within-subject evidence of FOSD violations. While Gneezy et al. (2006) report that they do not find the uncertainty effect in a withinsubject design, Sonsino (2008) shows evidence of within-subject violations. Specifically, 27 percent of his subjects value a binary lottery between two gift certificates below their own valuation of the lowest-valued (certain) prize paid by the lottery in at least one of their fifteen choices - although they are first reminded of their valuations of the certain outcomes. This results in an overall FOSD-violation rate of 12 percent. The FOSD-violation rate

\footnotetext{
${ }^{31}$ As we show in 3.2 the EBRD model is consistent with such effect.
} 
is about 20 percent when the probability of winning the better outcome is $0.1-0.3$, and about 4 percent when it is $0.8-0.9$ - consistent with the EBRD interpretation. 32 Finally, while the uncertainty-effect literature focused on FOSD violations with non-monetary prizes and deferred payments, Andreoni and Sprenger (2011) find violations with present monetary prizes. For example, using multiple price lists, 38 percent of their subjects reveal a preference for a certain monetary prize over a lottery with 95 percent of winning the same prize and 5 percent of winning a higher prize in at least one of three choices, with an overall violation rate of 17.5 percent 33 Sprenger (2015) finds a violation rate of 13.5 percent in a treatment identical to that of Andreoni and Sprenger (2011), but no violations in another treatment. We are not aware of other similar experiments.

There are, however, substantial and growing literatures on both preferences over compound lotteries (e.g., Spears, 2013) and preferences over the timing of non-instrumental information about uncertainty resolution (e.g., Bellemare et al., 2005; Masatlioglu et al., 2017). Such preferences necessarily violate dominance whenever they imply a strict willingness to pay for a preferred lottery structure or resolution timing. While we do not explore these complicated topics here, we note that settings with multi-stage resolution of uncertainty - where choices sometimes resemble "complexity-induced" violations (i.e., calculation mistakes) - are also exactly the types of settings that may induce a fear of disappointment of the type that drives our predictions here.

\section{Conclusion}

Our analysis in this paper suggests that at least some of the evidence of seemingly dominated choices in DA-like mechanisms, in both the field and the lab, may in fact reflect intentional behavior by expectations-based-loss-averse individuals. This conclusion invites a reinterpretation of said evidence. It also invites a shift in how we talk about behavior in DA-like mechanisms. Rather than expressing frustration over applicants' apparent failures

\footnotetext{
${ }^{32}$ Gneezy et al. (2006) elicit hypothetical WTP for $\$ 50$ and $\$ 100$ gift certificates, and a 50/50 lottery between the two, and find that 29/30 of subjects do not violate FOSD. Sonsino (2008) elicits incentivized WTP for gift certificates for different objects (a weekend vacation, dinner at a gourmet restaurant, and fine chocolate or wine), and for different lotteries that mix two of the three objects, with probabilities that range from 0.1 to 0.9. The EBRD model predicts both changes to increase observed FOSD violations. When asked later to explain their violations, 34 of Sonsino's subjects ( 54 percent) admit having exhibited them. Of the 34 subjects who admit violations, 22 (65 percent) choose "aversion to lotteries" as their preferred explanation, while 6 choose "noise or distraction" and another 6 choose "other explanations."

${ }^{33}$ In this experiment, a subject reports the value of $q$ that makes her indifferent between receiving $X$ (with certainty), and a lottery that pays $Y>X$ with probability $q$ and 0 otherwise, and the value of $q^{\prime}$ that makes her indifferent between the a lottery that pays $X$ with 95 percent, and $Y$ otherwise, and a lottery that pays $Y$ with probability $q^{\prime}$. Violations are identified when a subject reports $q>q^{\prime}$, indicating that she prefers receiving $X$ for sure over a lottery that pays $X$ with 95 percent, and $Y>X$ otherwise.
} 
to understand the mechanism, we might instead ask whether we have misspecified our model of preferences.

This conclusion also invites us to reconsider the advice we routinely give to applicants in DA-based matches. As mentioned in our introduction, the NRMP's website, for example, gives subjects the following advice: "To make the matching algorithm work best for you, create your rank-order list [hyphen added] in order of your true preferences, not how you think you will match." Such advice, if heeded by loss-averse applicants, may interfererather than help - with utility maximization: given applicants' concerns about disappointment, their perceived probability of matching with a program is anything but irrelevant. The evidence suggests that applicants in such mechanisms intentionally do not ignore this probability.

To complicate matters, however, we believe that this behavior, even if intentional, may normatively be a mistake. From a welfare point of view, even if individuals intentionally make choices whose immediate consequences they understand, and even though EBRD preferences are a real component of their well-being, there is reason to believe such preferences may reflect a mistaken overweighting of the gain-loss term in the utility function. In multi-round lab experiments, narrow bracketing (Benartzi and Thaler, 1999, Rabin and Weizsäcker, 2009) may make subjects fail to realize that gains and losses may offset each other across rounds. And even when subjects do think of multiple rounds jointly, non-belief in the law of large numbers (Benjamin et al., 2016) may still mean that they under-appreciate the extent of such offsetting. We speculate that in school choice, projection bias (Loewenstein et al., 2003) in particular may cause applicants to underestimate the speed with which they will recover from disappointment. Probably most importantly, in this context of DA, it seems very likely that people over-attend to gains and losses because they wrongly treat the immediate sensation as if it will last a long time. We emphasize, however, that even if the EBRD preferences we analyze in this paper are viewed as mistaken, the evidence suggests that their pursuit is often intentional, and not the result of people misunderstanding or miscalculating the immediate consequences of their choices.

We hope that future research will make progress on questions of stability, efficiency, and welfare analysis of different mechanisms under EBRD preferences. Ultimately, we hope that some of the relevant mechanisms may be redesigned in light of continuing analysis of the motives and preferences of people participating in those mechanisms. 


\section{References}

Abdulkadiroğlu, A., Pathak, P. A., and Roth, A. E. (2005). The New York City High School Match. American Economic Review, 95(2):364-367.

Andreoni, J. and Sprenger, C. (2011). Uncertainty Equivalents: Testing the Limits of the Independence Axiom. Working Paper 17342, National Bureau of Economic Research.

Artemov, G., Che, Y.-K., and He, Y. (2017). Strategic 'Mistakes': Implications for Market Design Research. Working paper.

Barberis, N. (2013). The Psychology of Tail Events: Progress and Challenges. American Economic Review, 103(3):611-16.

Bell, D. E. (1985). Disappointment in Decision Making under Uncertainty. Operations research, 33(1):1-27.

Bellemare, C., Krause, M., Kröger, S., and Zhang, C. (2005). Myopic loss aversion: Information feedback vs. investment flexibility. Economics Letters, 87(3):319-324.

Benartzi, S. and Thaler, R. H. (1999). Risk Aversion or Myopia? Choices in Repeated Gambles and Retirement Investments. Management Science, 45(3):364-381.

Benjamin, D. J., Heffetz, O., Kimball, M. S., and Rees-Jones, A. (2014). Can Marginal Rates of Substitution Be Inferred from Happiness Data? Evidence from Residency Choices. American Economic Review, 104(11):3498-3528.

Benjamin, D. J., Rabin, M., and Raymond, C. (2016). A model of nonbelief in the law of large numbers. Journal of the European Economic Association, 14(2):515-544.

Calsamiglia, C., Haeringer, G., and Klijn, F. (2010). Constrained School Choice: An Experimental Study. American Economic Review, 100(4):1860-74.

Chapman, J., Snowberg, E., Wang, S., and Camerer, C. (2018). Loss Attitudes in the U.S. Population: Evidence from Dynamically Optimized Sequential Experimentation (DOSE). Working Paper 25072, National Bureau of Economic Research.

Chen, Y. and Sönmez, T. (2006). School choice: an experimental study. Journal of Economic theory, 127(1):202-231.

Ding, T. and Schotter, A. (2017). Matching and chatting: An experimental study of the impact of network communication on school-matching mechanisms. Games and Economic Behavior, 103:94-115. 
Fack, G., Grenet, J., and He, Y. (2019). Beyond Truth-Telling: Preference Estimation with Centralized School Choice and College Admissions. American Economic Review, 109(4):1486-1529.

Fehr-Duda, H. and Epper, T. (2012). Probability and Risk: Foundations and Economic Implications of Probability-Dependent Risk Preferences. Annual Review of Economics, 4(1):567-593.

Gale, D. and Shapley, L. S. (1962). College Admissions and the Stability of Marriage. The American Mathematical Monthly, 69(1):9-15.

Gill, D. and Prowse, V. (2012). A Structural Analysis of Disappointment Aversion in a Real Effort Competition. American Economic Review, 102(1):469-503.

Gneezy, U., List, J. A., and Wu, G. (2006). The uncertainty effect: When a risky prospect is valued less than its worst possible outcome. The Quarterly Journal of Economics, 121(4):1283-1309.

Goette, L., Graeber, T., Kellogg, A., and Sprenger, C. (2018). Heterogeneity of Loss Aversion and Expectations-Based Reference Points. Available at SSRN: https://ssrn.com/abstract $=3170670$.

Guillen, P. and Hakimov, R. (2018). The effectiveness of top-down advice in strategy-proof mechanisms: A field experiment. European Economic Review, 101:505-511.

Guillen, P. and Hing, A. (2014). Lying through their teeth: Third party advice and truth telling in a strategy proof mechanism. European Economic Review, 70:178-185.

Gul, F. (1991). A Theory of Disappointment Aversion. Econometrica, 59(3):667-686.

Hakimov, R. and Kübler, D. (2019). Experiments On Matching Markets: A Survey. Working paper.

Hassidim, A., Romm, A., and Shorrer, R. I. (2018). 'Strategic' Behavior in a Strategy-Proof Environment. Available at SSRN: https://ssrn.com/abstract=2784659.

Kahneman, D. and Tversky, A. (1979). Prospect Theory: An Analysis of Decision under Risk. Econometrica, 47(2):263-291.

Keren, G. and Willemsen, M. C. (2009). Decision anomalies, experimenter assumptions, and participants' comprehension: Revaluating the uncertainty effect. Journal of Behavioral Decision Making, 22(3):301-317. 
Klijn, F., Pais, J., and Vorsatz, M. (2013). Preference intensities and risk aversion in school choice: A laboratory experiment. Experimental Economics, 16(1):1-22.

Köszegi, B. (2006). Ego utility, overconfidence, and task choice. Journal of the European Economic Association, 4(4):673-707.

Köszegi, B. and Rabin, M. (2006). A Model of Reference-Dependent Preferences. The Quarterly Journal of Economics, 121(4):1133-1165.

Kőszegi, B. and Rabin, M. (2007). Reference-Dependent Risk Attitudes. American Economic Review, 97(4):1047-1073.

Kőszegi, B. and Rabin, M. (2009). Reference-Dependent Consumption Plans. American Economic Review, 99(3):909-36.

Li, S. (2017). Obviously Strategy-Proof Mechanisms. American Economic Review, 107(11):3257-87.

Loewenstein, G., O’Donoghue, T., and Rabin, M. (2003). Projection Bias in Predicting Future Utility. The Quarterly Journal of Economics, 118(4):1209-1248.

Loomes, G. and Sugden, R. (1986). Disappointment and Dynamic Consistency in Choice under Uncertainty. The Review of Economic Studies, 53(2):271-282.

Masatlioglu, Y., Orhun, A. Y., and Raymond, C. (2017). Intrinsic Information Preferences and Skewness. Available at SSRN: https://ssrn.com/abstract=3232350.

McKelvey, R. D. and Palfrey, T. R. (1995). Quantal Response Equilibria for Normal Form Games. Games and Economic Behavior, 10(1):6-38.

Rabin, M. and Weizsäcker, G. (2009). Narrow Bracketing and Dominated Choices. American Economic Review, 99(4):1508-43.

Rees-Jones, A. (2018). Suboptimal behavior in strategy-proof mechanisms: Evidence from the residency match. Games and Economic Behavior, 108:317-330.

Rees-Jones, A., Shorrer, R. I., and Tergiman, C. (2019). Correlation Neglect in Student-toSchool Matching. Available at SSRN: https://ssrn.com/abstract=3434662.

Rees-Jones, A. and Skowronek, S. (2018). An experimental investigation of preference misrepresentation in the residency match. Proceedings of the National Academy of Sciences, 115(45):11471-11476. 
Roth, A. E. and Peranson, E. (1999). The Redesign of the Matching Market for American Physicians: Some Engineering Aspects of Economic Design. American Economic Review, 89(4):748-780.

Rydval, O., Ortmann, A., Prokosheva, S., and Hertwig, R. (2009). How certain is the uncertainty effect? Experimental Economics, 12(4):473-487.

Shorrer, R. I. and Sóvágó, S. (2018). Obvious Mistakes in a Strategically Simple College Admissions Environment: Causes and Consequences. Available at SSRN: https://ssrn.com/abstract $=2993538$.

Simonsohn, U. (2009). Direct Risk Aversion: Evidence From Risky Prospects Valued Below Their Worst Outcome. Psychological Science, 20(6):686-692.

Sonsino, D. (2008). Disappointment aversion in internet bidding-decisions. Theory and Decision, 64(2-3):363-393.

Spears, D. (2013). Poverty and probability: aspiration and aversion to compound lotteries in El Salvador and India. Experimental Economics, 16(3):263-284.

Sprenger, C. (2015). An Endowment Effect for Risk: Experimental Tests of Stochastic Reference Points. Journal of Political Economy, 123(6):1456-1499.

Train, K. E. (2009). Discrete Choice Methods with Simulation. Cambridge University Press, 2 edition. 


\section{Web Appendix}

\section{A Prior Expectations}

\section{A.1 Two Schools}

In this section we relax the assumption that Lori's inherited beliefs are 0 in all dimensions, and examine other possible prior expectations. We consider three possible beliefs entering submission period: (a) Lori expected to attend $s_{1}$ (with probability 1); (b) Lori expected to attend $s_{2}$ (with probability 1 ); and (c) Lori expected the lottery generated by ranking truthfully (that is, attending $s_{1}$ with probability $q_{1}$ and $s_{2}$ with probability $\left(1-q_{1}\right) q_{2}$ ).

Table A.1: Utility with Prior Expectations

\begin{tabular}{|c|c|c|c|c|}
\hline \multirow{2}{*}{ Submission Utility } & \multicolumn{4}{|c|}{ Prior Expectations } \\
\hline & $\begin{array}{c}\left(s_{1}, 0 ; s_{2}, 0\right) \\
\text { (Baseline) }\end{array}$ & $\begin{array}{c}\left(s_{1}, 1\right) \\
\left(s_{1} \text { for sure }\right)\end{array}$ & $\begin{array}{c}\left(s_{2}, 1\right) \\
\left(s_{2} \text { for sure }\right)\end{array}$ & $\begin{array}{l}L\left(s_{1} \widehat{\succ} s_{2}\right) \\
\text { (Truthful) }\end{array}$ \\
\hline $\begin{array}{l}u_{1}\left(L\left(s_{1} \widehat{\succ} s_{2}\right)\right) \\
u_{1}\left(L\left(s_{2} \widehat{\succ} s_{1}\right)\right) \\
u_{1}\left(L\left(s_{1}\right)\right) \\
u_{1}\left(L\left(s_{2}\right)\right) \\
u_{1}(L(\emptyset))\end{array}$ & $\begin{array}{c}q_{1} m_{1}+\left(1-q_{1}\right) q_{2} m_{2} \\
\left(1-q_{2}\right) q_{1} m_{1}+q_{2} m_{2} \\
q_{1} m_{1} \\
q_{2} m_{2} \\
0\end{array}$ & $\begin{array}{c}-\lambda\left(1-q_{1}\right) m_{1}+\left(1-q_{1}\right) q_{2} m_{2} \\
-\lambda\left(1-\left(1-q_{2}\right) q_{1}\right) m_{1}+q_{2} m_{2} \\
-\lambda\left(1-q_{1}\right) m_{1} \\
-\lambda m_{1}+q_{2} m_{2} \\
-\lambda m_{1}\end{array}$ & $\begin{array}{c}q_{1} m_{1}-\lambda\left(1-\left(1-q_{1}\right) q_{2}\right) m_{2} \\
\left(1-q_{2}\right) q_{1} m_{1}-\lambda\left(1-q_{2}\right) m_{2} \\
q_{1} m_{1}-\lambda m_{2} \\
-\lambda\left(1-q_{2}\right) m_{2} \\
-\lambda m_{2} \\
\end{array}$ & $\begin{array}{c}0 \\
-\lambda q_{1} q_{2} m_{1}+q_{2} q_{1} m_{2} \\
-\lambda\left(1-q_{1}\right) q_{2} m_{2} \\
-\lambda q_{1}+q_{1} q_{2} m_{2} \\
-\lambda q_{1} m_{1}-\lambda\left(1-q_{1}\right) q_{2} m_{2}\end{array}$ \\
\hline
\end{tabular}

Notes: Period-1 news utility from submitting different ROLs, given different prior expectations.

Table A.1 shows period-1 news utility from all possible ROLs, given different prior expectations. In general, period-1 utility in each dimension $i$ in this case is given by

$$
u_{1}^{i}(p \mid \hat{p})=\left\{\begin{array}{ll}
\left(p_{i}-\hat{p}_{i}\right) m_{i} & p_{i} \geq \hat{p}_{i} \\
-\lambda\left(\hat{p}_{i}-p\right) m_{i} & \hat{p}_{i}>p_{i}
\end{array},\right.
$$

where $p_{i}$ denotes as usual the ROL-determined probability of attending $s_{i}$, and $\hat{p}_{i}$ denotes the probability Lori assigned to attending $s_{i}$ entering period 1. Since Lori updates her beliefs according to her submitted ROL, period-2 utility is not affected by prior expectations. Of course, period-3 consumption utility is not affected by prior beliefs either.

When Lori enters period 1 expecting to attend a school $s_{i}$ with probability 1 (that is, when $\hat{p}_{i}=1$ ), the incentive to omit $s_{i}$ from the ROL completely vanishes. To see why, 
observe that for any $p_{i}, \lambda m_{i}>\lambda\left(1-p_{i}\right) m_{i}+(\lambda-1) p_{i}\left(1-p_{i}\right) m_{i}$. The LHS of this inequality is the cost of omitting $s_{i}$, and the RHS is the cost of including it, both assuming $\hat{p}_{i}=1$. Using similar arguments, we can show that the same is true for both schools when prior expectations are the lottery generated by the submission of a truthful list.

Figures A.1 A.3 show the model's predicted submission for various values of $q_{1}, q_{2}, m$ and $\lambda$.

Figure A.1: Theoretical Predictions (Prior $\left(s_{1}, 1\right)$ )
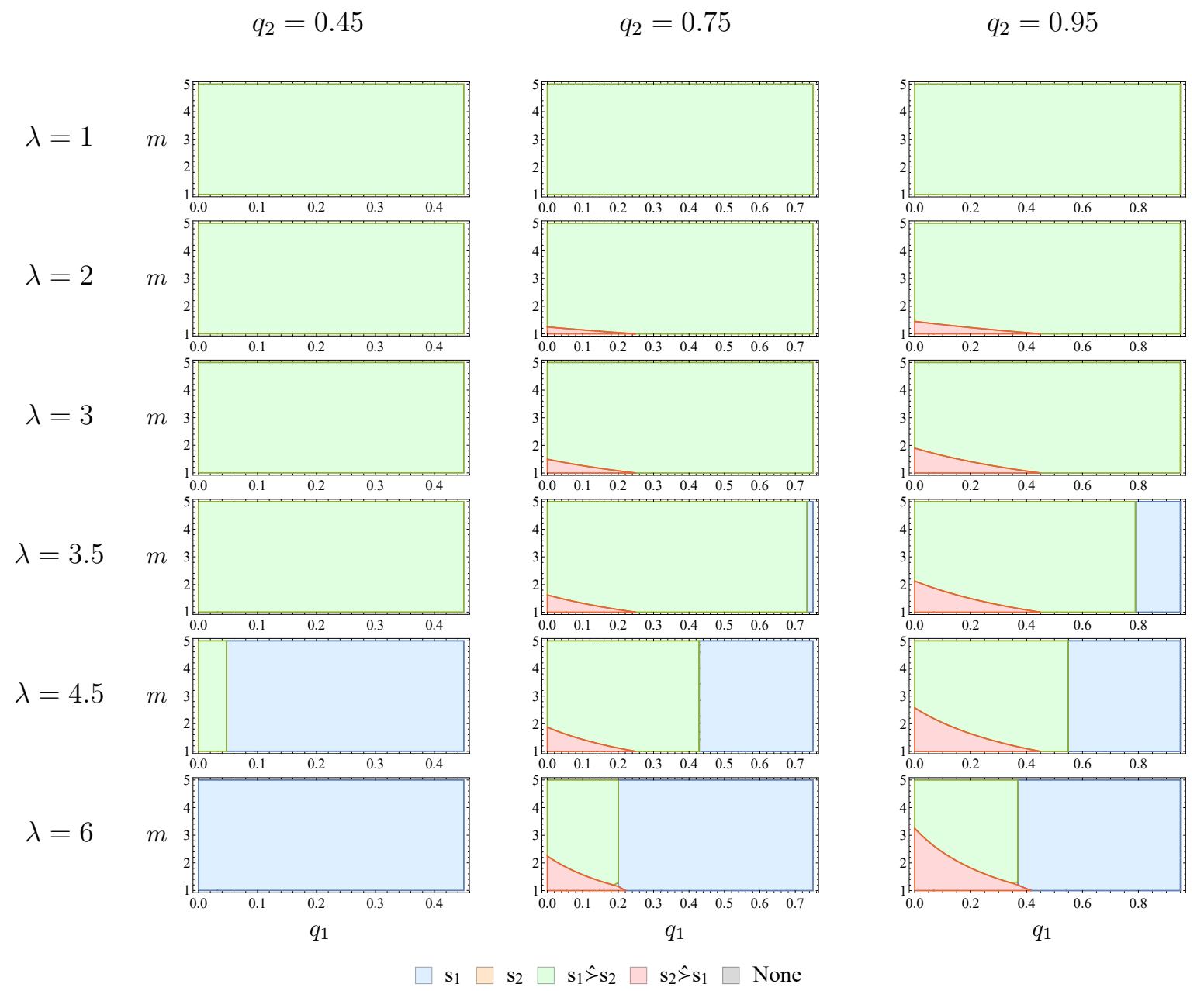

Notes: Model-predicted submitted list as a function of the model's parameters: each subplot shows the model's predictions for combinations of $q_{1}$ and $m$, fixing $q_{2}$ and $\lambda$. " $s_{1}$ " and " $s_{2}$ " denote lists containing only one school, with the other school omitted ( $s_{1}$ is the preferred school). "None" denotes an empty list. Prior expectations: $s_{1}$ with certainty.

\section{A.2 Funded Positions}

We now examine the effect of prior expectations on observed violations of FOSD (obvious misrepresentations). Let $\hat{p}$ be the probability that Lori assigns to getting a funded position 
Figure A.2: Theoretical Predictions (Prior $\left(s_{2}, 1\right)$ )

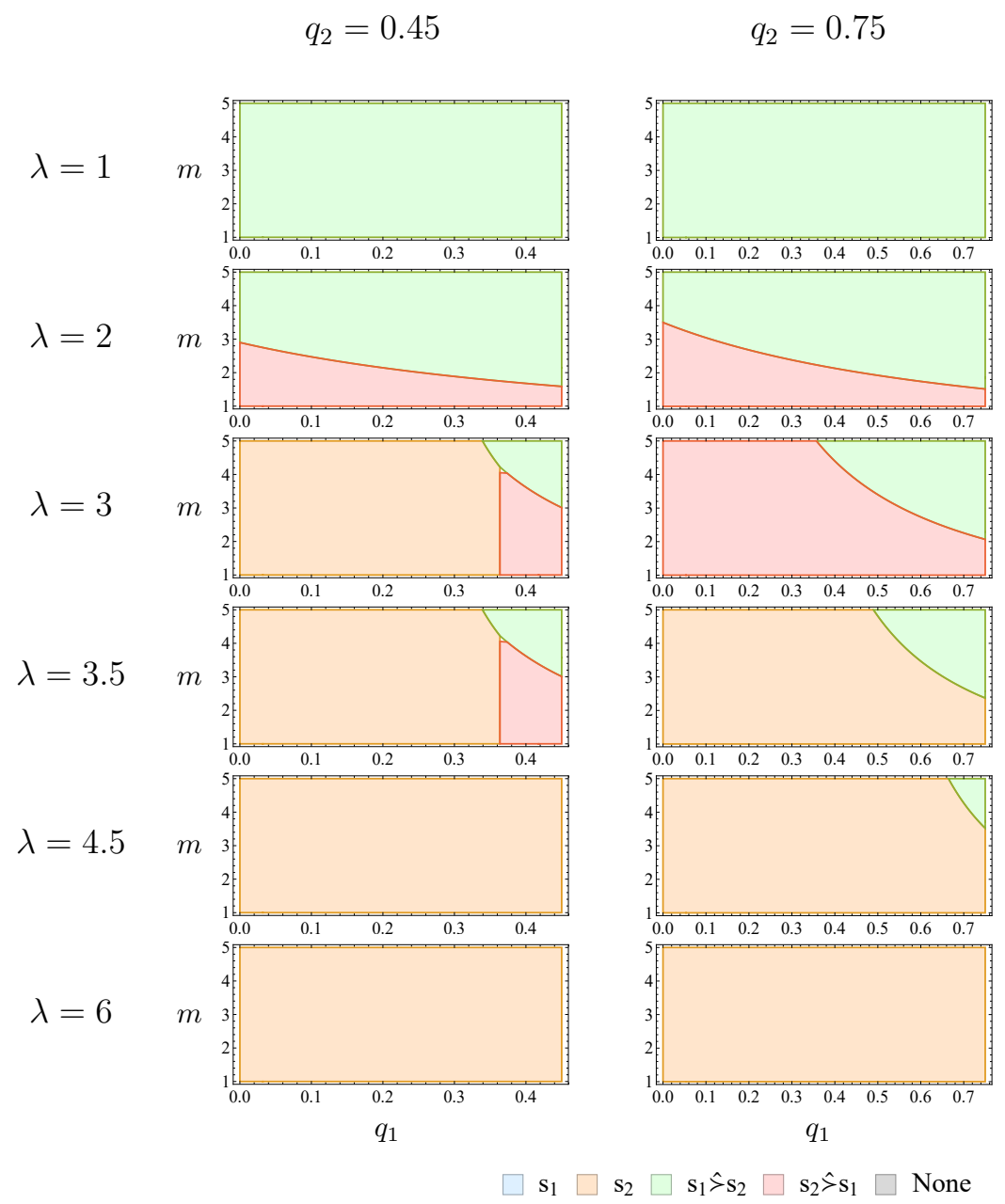

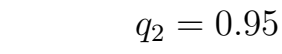
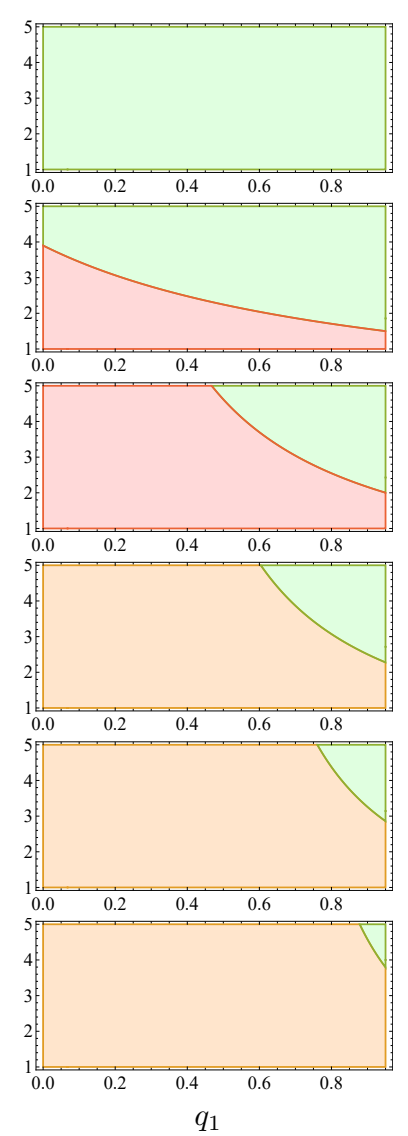

Notes: Model-predicted submitted list as a function of the model's parameters: each subplot shows the model's predictions for combinations of $q_{1}$ and $m$, fixing $q_{2}$ and $\lambda$. " $s_{1}$ " and " $s_{2}$ " denote lists containing only one school, with the other school omitted ( $s_{1}$ is the preferred school). "None" denotes an empty list. Prior expectations: $s_{2}$ with certainty.

entering period 1. The amount of funding is given by $\$ x$. In period 1 Lori learns that if she ranks the funded position truthfully, she will get it with probability $p$. For simplicity, we focus on the omission versus ranking truthfully only. First, if Lori is overoptimistic, that is, if $\hat{p}>p$, omission is preferred to ranking truthfully iff

$$
-\lambda \hat{p} m^{\text {money }}(x)>-\lambda(\hat{p}-p) m^{\text {money }}-(\lambda-1) p(1-p) m^{\text {money }}+p m^{\text {money }} .
$$

It is straightforward to see that this inequality never holds, so Lori is never predicted to omit funding in this case.

Second, if Lori is pessimistic (or otherwise is less aware of funding prior to the submission 
Figure A.3: Theoretical Predictions (Prior $L\left(s_{1} \widehat{\succ} s_{2}\right)$ )

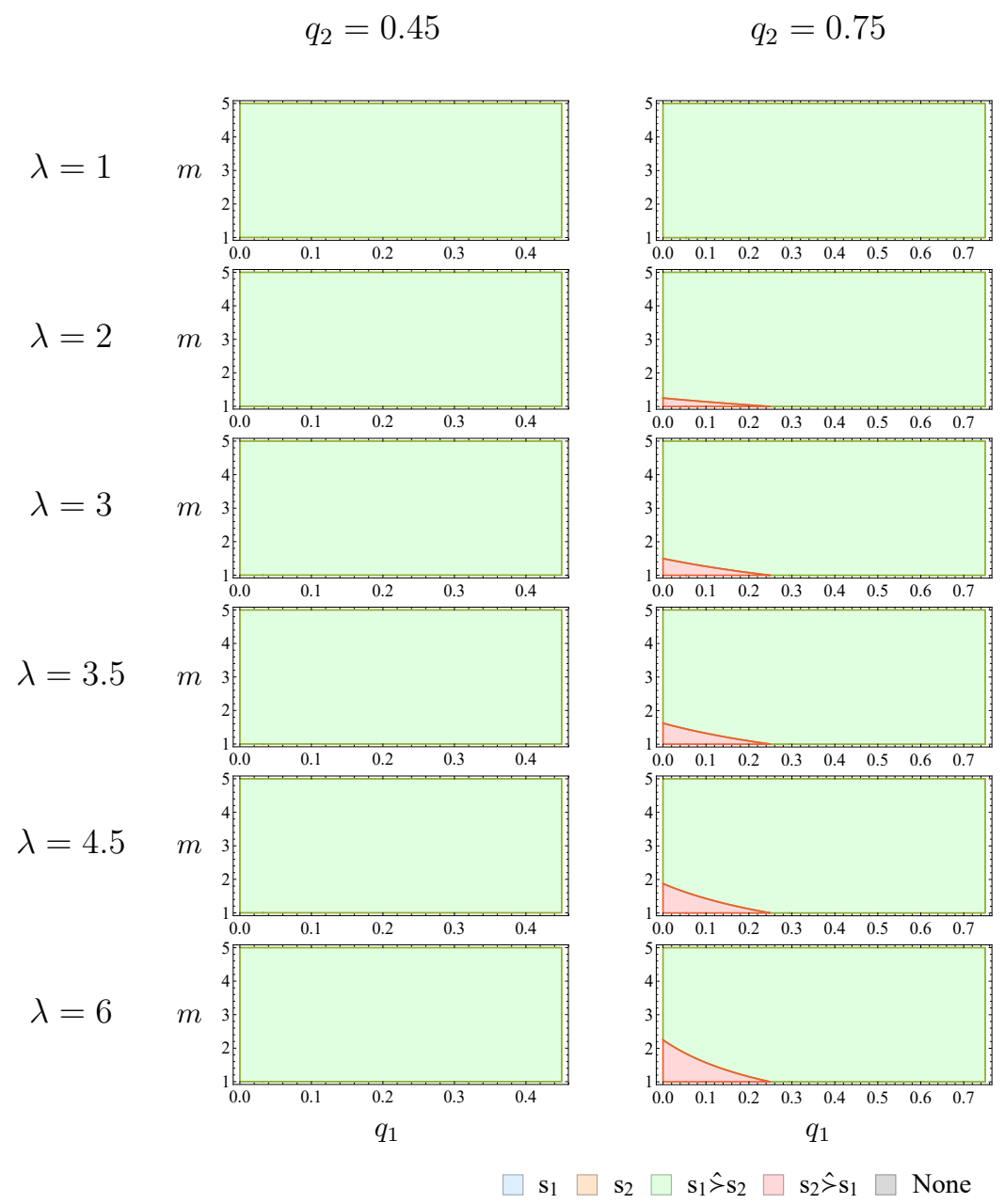

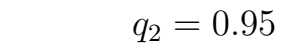
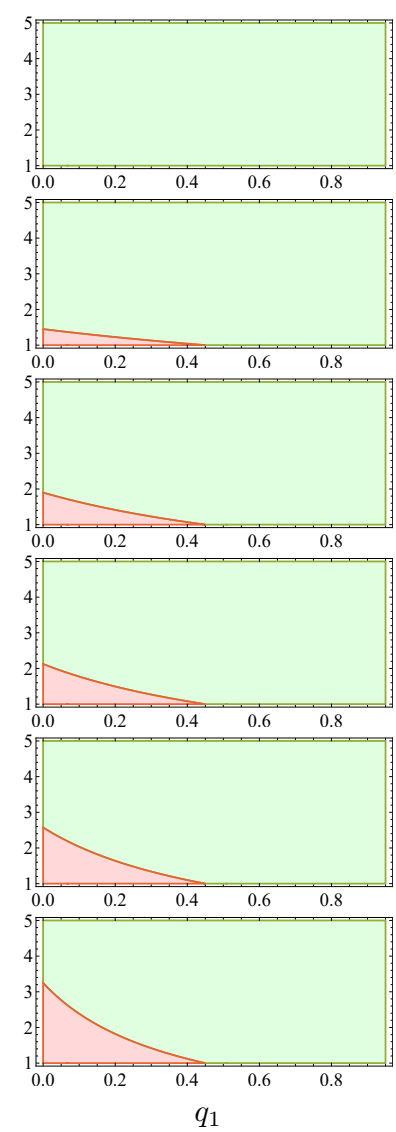

Notes: Model-predicted submitted list as a function of the model's parameters: each subplot shows the model's predictions for combinations of $q_{1}$ and $m$, fixing $q_{2}$ and $\lambda$. " $s_{1}$ " and " $s_{2}$ " denote lists containing only one school, with the other school omitted ( $s_{1}$ is the preferred school). "None" denotes an empty list. Prior expectations: the lottery generated by the submission of a truthful list.

period), or $\hat{p}<p$, then she prefers to omit over ranking truthfully iff

$$
-\lambda \hat{p} m^{\text {money }}(x)>(p-\hat{p}) m^{\text {money }}-(\lambda-1) p(1-p) m^{\text {money }}+p m^{\text {money }}
$$

which reduces to

$$
\hat{p}<p\left(1-p-\frac{2}{\lambda-1}\right),
$$

which means that if Lori enters period 1 with enough pessimism about her chances of receiving funding, and if she is sufficiently loss averse, she is predicted to obviously misrepresent. 


\section{B Instructions (Reproduced From Li, 2017)}

\section{GAME 3}

You will play this game for 10 rounds. In each round of this game, there are four prizes, labeled A, B, C, and D. Prizes will be worth between $\$ 0.00$ and $\$ 1.25$. For each prize, its value will be the same for all the players in your group.

At the start of each round, you will learn the value of each prize. You will also learn your priority score, which is a random number between 1 and 10 . Every whole number between 1 and 10 is equally likely to be chosen.

The game proceeds as follows: We will ask you to list the prizes, in any order of your choice. All players will submit their lists privately and at the same time.

After all the lists have been submitted, we will assign prizes using the following rule:

1. The player with the highest priority score will be assigned the top prize on his list.

2. The player with the second-highest priority score will be assigned the top prize on his list, among the prizes that remain.

3. The player with the third-highest priority score will be assigned the top prize on his list, among the prizes that remain.

4. The player with the lowest priority score will be assigned whatever prize remains.

If two players have the same priority score, we will break the tie randomly.

You will have 90 seconds to form your list. You do this by typing a number, from 1 to 4 , next to each prize, and then clicking the button that says "Confirm Choices". Each prize must be assigned a different number, from 1 (top) to 4 (bottom). Your choices will not count unless you click the button that says "Confirm Choices".

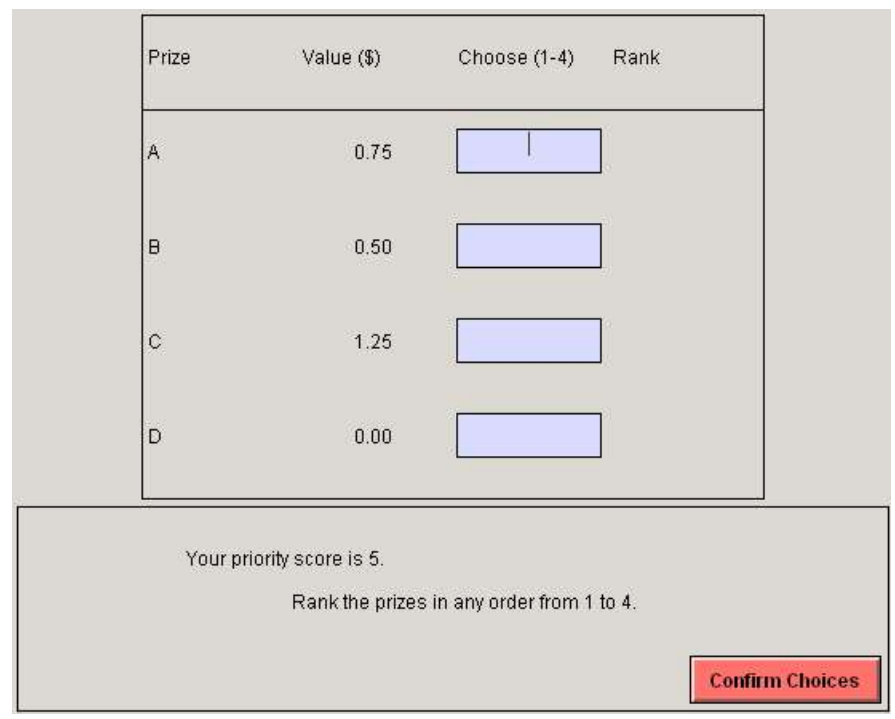

5 of 6 
If you do not produce a list by the end of 90 seconds, we will assign prizes as though you reported the list in order A-B-C-D.

At the end of each round, we will add to your earnings the value of the prize you were assigned. 


\section{Raw Distribution of Lists}

Table C.1: Empirical Distribution of Eight List Sets (Multiround, Misrepresenters Only)

\begin{tabular}{lccccccccccc}
\hline \hline ROLs & \#ROLs & & \multicolumn{1}{c}{ Priority Score } & & & & \\
& & 1 & 2 & 3 & 4 & 5 & 6 & 7 & 8 & 9 & 10 \\
\hline & & & & & & & & & & & \\
1234 & 1 & $34.0 \%$ & $37.9 \%$ & $29.8 \%$ & $44.4 \%$ & $33.3 \%$ & $59.4 \%$ & $60.0 \%$ & $75.0 \%$ & $73.8 \%$ & $86.3 \%$ \\
1243 & 1 & $1.9 \%$ & $1.7 \%$ & $2.1 \%$ & $0.0 \%$ & $0.0 \%$ & $3.1 \%$ & $2.0 \%$ & $0.0 \%$ & $2.4 \%$ & $0.0 \%$ \\
$13 \mathrm{XX}$ & 2 & $5.7 \%$ & $5.2 \%$ & $4.3 \%$ & $2.8 \%$ & $7.7 \%$ & $0.0 \%$ & $2.0 \%$ & $0.0 \%$ & $2.4 \%$ & $2.0 \%$ \\
$14 \mathrm{XX}$ & 2 & $0.0 \%$ & $1.7 \%$ & $0.0 \%$ & $2.8 \%$ & $2.6 \%$ & $0.0 \%$ & $0.0 \%$ & $0.0 \%$ & $0.0 \%$ & $2.0 \%$ \\
$2^{*} 3^{*} 4^{*}$ & 3 & $3.8 \%$ & $5.2 \%$ & $10.6 \%$ & $19.4 \%$ & $23.1 \%$ & $18.8 \%$ & $16.0 \%$ & $18.8 \%$ & $14.3 \%$ & $3.9 \%$ \\
$2^{*} 4^{*} 3^{*}$ & 3 & $0.0 \%$ & $3.4 \%$ & $10.6 \%$ & $0.0 \%$ & $2.6 \%$ & $3.1 \%$ & $4.0 \%$ & $0.0 \%$ & $0.0 \%$ & $2.0 \%$ \\
3 XXX & 6 & $18.9 \%$ & $15.5 \%$ & $21.3 \%$ & $11.1 \%$ & $17.9 \%$ & $0.0 \%$ & $10.0 \%$ & $3.1 \%$ & $2.4 \%$ & $0.0 \%$ \\
4 XXX & 6 & $35.8 \%$ & $29.3 \%$ & $21.3 \%$ & $19.4 \%$ & $12.8 \%$ & $15.6 \%$ & $6.0 \%$ & $3.1 \%$ & $4.8 \%$ & $3.9 \%$ \\
\hline Total & 24 & $100.0 \%$ & $100.0 \%$ & $100.0 \%$ & $100.0 \%$ & $100.0 \%$ & $100.0 \%$ & $100.0 \%$ & $100.0 \%$ & $100.0 \%$ & $100.0 \%$ \\
\hline$N$ & & 53 & 58 & 47 & 36 & 39 & 32 & 50 & 32 & 42 & 51 \\
\hline
\end{tabular}

Notes: Share of decisions as a percentage of choice situations with the same priority score. ROLs are grouped into sets as explained in figure 4 a

Table C.2: Empirical Distribution of Eight List Sets (One Shot)

\begin{tabular}{lccccccccccc}
\hline \hline ROLs & \#ROLs & & \multicolumn{1}{c}{ Priority Score } & & & & \\
& & 1 & 2 & 3 & 4 & 5 & 6 & 7 & 8 & 9 & 10 \\
\hline & & & & & & & & & & & \\
1234 & 1 & $92.3 \%$ & $26.7 \%$ & $69.6 \%$ & $47.8 \%$ & $71.4 \%$ & $47.1 \%$ & $55.0 \%$ & $87.5 \%$ & $81.8 \%$ & $81.0 \%$ \\
1243 & 1 & $0.0 \%$ & $0.0 \%$ & $0.0 \%$ & $0.0 \%$ & $0.0 \%$ & $0.0 \%$ & $5.0 \%$ & $0.0 \%$ & $0.0 \%$ & $0.0 \%$ \\
$13 X X$ & 2 & $0.0 \%$ & $0.0 \%$ & $0.0 \%$ & $0.0 \%$ & $0.0 \%$ & $5.9 \%$ & $0.0 \%$ & $0.0 \%$ & $0.0 \%$ & $9.5 \%$ \\
14 XX & 2 & $0.0 \%$ & $0.0 \%$ & $0.0 \%$ & $0.0 \%$ & $0.0 \%$ & $0.0 \%$ & $0.0 \%$ & $0.0 \%$ & $0.0 \%$ & $0.0 \%$ \\
$2^{*} 3^{*} 4^{*}$ & 3 & $7.7 \%$ & $33.3 \%$ & $8.7 \%$ & $21.7 \%$ & $21.4 \%$ & $23.5 \%$ & $25.0 \%$ & $4.2 \%$ & $4.5 \%$ & $0.0 \%$ \\
$2^{*} 4^{*} 3^{*}$ & 3 & $0.0 \%$ & $0.0 \%$ & $0.0 \%$ & $4.3 \%$ & $0.0 \%$ & $0.0 \%$ & $5.0 \%$ & $0.0 \%$ & $4.5 \%$ & $0.0 \%$ \\
3 XXX & 6 & $0.0 \%$ & $13.3 \%$ & $21.7 \%$ & $13.0 \%$ & $0.0 \%$ & $17.6 \%$ & $0.0 \%$ & $0.0 \%$ & $0.0 \%$ & $9.5 \%$ \\
4 XXX & 6 & $0.0 \%$ & $26.7 \%$ & $0.0 \%$ & $13.0 \%$ & $7.1 \%$ & $5.9 \%$ & $10.0 \%$ & $8.3 \%$ & $9.1 \%$ & $0.0 \%$ \\
\hline Total & 24 & $100.0 \%$ & $100.0 \%$ & $100.0 \%$ & $100.0 \%$ & $100.0 \%$ & $100.0 \%$ & $100.0 \%$ & $100.0 \%$ & $100.0 \%$ & $100.0 \%$ \\
\hline$N$ & & 13 & 15 & 23 & 23 & 14 & 17 & 20 & 24 & 22 & 21 \\
\hline
\end{tabular}

Notes: Share of decisions as a percentage of choice situations with the same priority score. ROLs are grouped into sets as explained in figure 4 a 
Table C.3: Empirical Distribution of Lists (Multiround)

\begin{tabular}{|c|c|c|c|c|c|c|c|c|c|c|}
\hline \multirow[t]{2}{*}{$\mathrm{ROL}$} & \multicolumn{10}{|c|}{ Priority Score } \\
\hline & 1 & 2 & 3 & 4 & 5 & 6 & 7 & 8 & 9 & 10 \\
\hline 1234 & $61.1 \%$ & $57.1 \%$ & $58.8 \%$ & $67.7 \%$ & $55.2 \%$ & $79.0 \%$ & $74.4 \%$ & $85.7 \%$ & $84.3 \%$ & $91.3 \%$ \\
\hline 1243 & $1.1 \%$ & $1.2 \%$ & $1.3 \%$ & $0.0 \%$ & $0.0 \%$ & $1.6 \%$ & $1.3 \%$ & $0.0 \%$ & $1.4 \%$ & $0.0 \%$ \\
\hline 1324 & $2.2 \%$ & $3.6 \%$ & $2.5 \%$ & $1.6 \%$ & $3.4 \%$ & $0.0 \%$ & $1.3 \%$ & $0.0 \%$ & $1.4 \%$ & $0.0 \%$ \\
\hline 1342 & $1.1 \%$ & $0.0 \%$ & $0.0 \%$ & $0.0 \%$ & $1.7 \%$ & $0.0 \%$ & $0.0 \%$ & $0.0 \%$ & $0.0 \%$ & $1.3 \%$ \\
\hline 1423 & $0.0 \%$ & $1.2 \%$ & $0.0 \%$ & $1.6 \%$ & $1.7 \%$ & $0.0 \%$ & $0.0 \%$ & $0.0 \%$ & $0.0 \%$ & $0.0 \%$ \\
\hline 1432 & $0.0 \%$ & $0.0 \%$ & $0.0 \%$ & $0.0 \%$ & $0.0 \%$ & $0.0 \%$ & $0.0 \%$ & $0.0 \%$ & $0.0 \%$ & $1.3 \%$ \\
\hline 2134 & $1.1 \%$ & $1.2 \%$ & $3.8 \%$ & $6.5 \%$ & $12.1 \%$ & $8.1 \%$ & $10.3 \%$ & $7.1 \%$ & $5.7 \%$ & $1.3 \%$ \\
\hline 2143 & $0.0 \%$ & $1.2 \%$ & $3.8 \%$ & $0.0 \%$ & $1.7 \%$ & $0.0 \%$ & $0.0 \%$ & $0.0 \%$ & $0.0 \%$ & $1.3 \%$ \\
\hline 2314 & $1.1 \%$ & $2.4 \%$ & $2.5 \%$ & $1.6 \%$ & $3.4 \%$ & $1.6 \%$ & $0.0 \%$ & $3.6 \%$ & $1.4 \%$ & $1.3 \%$ \\
\hline 2341 & $0.0 \%$ & $0.0 \%$ & $0.0 \%$ & $3.2 \%$ & $0.0 \%$ & $0.0 \%$ & $0.0 \%$ & $0.0 \%$ & $1.4 \%$ & $0.0 \%$ \\
\hline 2413 & $0.0 \%$ & $1.2 \%$ & $2.5 \%$ & $0.0 \%$ & $0.0 \%$ & $0.0 \%$ & $2.6 \%$ & $0.0 \%$ & $0.0 \%$ & $0.0 \%$ \\
\hline 2431 & $0.0 \%$ & $0.0 \%$ & $0.0 \%$ & $0.0 \%$ & $0.0 \%$ & $1.6 \%$ & $0.0 \%$ & $0.0 \%$ & $0.0 \%$ & $0.0 \%$ \\
\hline 3124 & $1.1 \%$ & $2.4 \%$ & $2.5 \%$ & $1.6 \%$ & $5.2 \%$ & $0.0 \%$ & $5.1 \%$ & $0.0 \%$ & $1.4 \%$ & $0.0 \%$ \\
\hline 3142 & $0.0 \%$ & $0.0 \%$ & $0.0 \%$ & $0.0 \%$ & $0.0 \%$ & $0.0 \%$ & $0.0 \%$ & $0.0 \%$ & $0.0 \%$ & $0.0 \%$ \\
\hline 3214 & $6.7 \%$ & $6.0 \%$ & $7.5 \%$ & $4.8 \%$ & $3.4 \%$ & $0.0 \%$ & $0.0 \%$ & $1.8 \%$ & $0.0 \%$ & $0.0 \%$ \\
\hline 3241 & $0.0 \%$ & $0.0 \%$ & $1.3 \%$ & $0.0 \%$ & $0.0 \%$ & $0.0 \%$ & $1.3 \%$ & $0.0 \%$ & $0.0 \%$ & $0.0 \%$ \\
\hline 3412 & $0.0 \%$ & $0.0 \%$ & $1.3 \%$ & $0.0 \%$ & $3.4 \%$ & $0.0 \%$ & $0.0 \%$ & $0.0 \%$ & $0.0 \%$ & $0.0 \%$ \\
\hline 3421 & $3.3 \%$ & $2.4 \%$ & $0.0 \%$ & $0.0 \%$ & $0.0 \%$ & $0.0 \%$ & $0.0 \%$ & $0.0 \%$ & $0.0 \%$ & $0.0 \%$ \\
\hline 4123 & $1.1 \%$ & $2.4 \%$ & $1.3 \%$ & $0.0 \%$ & $1.7 \%$ & $3.2 \%$ & $1.3 \%$ & $0.0 \%$ & $0.0 \%$ & $1.3 \%$ \\
\hline 4132 & $0.0 \%$ & $1.2 \%$ & $0.0 \%$ & $0.0 \%$ & $0.0 \%$ & $0.0 \%$ & $0.0 \%$ & $0.0 \%$ & $0.0 \%$ & $0.0 \%$ \\
\hline 4213 & $1.1 \%$ & $1.2 \%$ & $0.0 \%$ & $1.6 \%$ & $5.2 \%$ & $1.6 \%$ & $1.3 \%$ & $0.0 \%$ & $0.0 \%$ & $0.0 \%$ \\
\hline 4231 & $1.1 \%$ & $2.4 \%$ & $2.5 \%$ & $0.0 \%$ & $0.0 \%$ & $0.0 \%$ & $0.0 \%$ & $1.8 \%$ & $0.0 \%$ & $0.0 \%$ \\
\hline 4312 & $0.0 \%$ & $4.8 \%$ & $5.0 \%$ & $4.8 \%$ & $0.0 \%$ & $0.0 \%$ & $0.0 \%$ & $0.0 \%$ & $0.0 \%$ & $1.3 \%$ \\
\hline 4321 & $17.8 \%$ & $8.3 \%$ & $3.8 \%$ & $4.8 \%$ & $1.7 \%$ & $3.2 \%$ & $1.3 \%$ & $0.0 \%$ & $2.9 \%$ & $0.0 \%$ \\
\hline Total & $100.0 \%$ & $100.0 \%$ & $100.0 \%$ & $100.0 \%$ & $100.0 \%$ & $100.0 \%$ & $100.0 \%$ & $100.0 \%$ & $100.0 \%$ & $100.0 \%$ \\
\hline$N$ & 90 & 84 & 80 & 62 & 58 & 62 & 78 & 56 & 70 & 80 \\
\hline
\end{tabular}

Note: Share of decisions as a percentage of choice situations with the same priority score. 
Table C.4: Empirical Distribution of Lists (Multiround, Misrepresenters Only)

\begin{tabular}{|c|c|c|c|c|c|c|c|c|c|c|}
\hline \multirow[t]{2}{*}{ ROL } & \multicolumn{10}{|c|}{ Priority Score } \\
\hline & 1 & 2 & 3 & 4 & 5 & 6 & 7 & 8 & 9 & 10 \\
\hline 1234 & $34.0 \%$ & $37.9 \%$ & $29.8 \%$ & $44.4 \%$ & $33.3 \%$ & $59.4 \%$ & $60.0 \%$ & $75.0 \%$ & $73.8 \%$ & $86.3 \%$ \\
\hline 1243 & $1.9 \%$ & $1.7 \%$ & $2.1 \%$ & $0.0 \%$ & $0.0 \%$ & $3.1 \%$ & $2.0 \%$ & $0.0 \%$ & $2.4 \%$ & $0.0 \%$ \\
\hline 1324 & $3.8 \%$ & $5.2 \%$ & $4.3 \%$ & $2.8 \%$ & $5.1 \%$ & $0.0 \%$ & $2.0 \%$ & $0.0 \%$ & $2.4 \%$ & $0.0 \%$ \\
\hline 1342 & $1.9 \%$ & $0.0 \%$ & $0.0 \%$ & $0.0 \%$ & $2.6 \%$ & $0.0 \%$ & $0.0 \%$ & $0.0 \%$ & $0.0 \%$ & $2.0 \%$ \\
\hline 1423 & $0.0 \%$ & $1.7 \%$ & $0.0 \%$ & $2.8 \%$ & $2.6 \%$ & $0.0 \%$ & $0.0 \%$ & $0.0 \%$ & $0.0 \%$ & $0.0 \%$ \\
\hline 1432 & $0.0 \%$ & $0.0 \%$ & $0.0 \%$ & $0.0 \%$ & $0.0 \%$ & $0.0 \%$ & $0.0 \%$ & $0.0 \%$ & $0.0 \%$ & $2.0 \%$ \\
\hline 2134 & $1.9 \%$ & $1.7 \%$ & $6.4 \%$ & $11.1 \%$ & $17.9 \%$ & $15.6 \%$ & $16.0 \%$ & $12.5 \%$ & $9.5 \%$ & $2.0 \%$ \\
\hline 2143 & $0.0 \%$ & $1.7 \%$ & $6.4 \%$ & $0.0 \%$ & $2.6 \%$ & $0.0 \%$ & $0.0 \%$ & $0.0 \%$ & $0.0 \%$ & $2.0 \%$ \\
\hline 2314 & $1.9 \%$ & $3.4 \%$ & $4.3 \%$ & $2.8 \%$ & $5.1 \%$ & $3.1 \%$ & $0.0 \%$ & $6.3 \%$ & $2.4 \%$ & $2.0 \%$ \\
\hline 2341 & $0.0 \%$ & $0.0 \%$ & $0.0 \%$ & $5.6 \%$ & $0.0 \%$ & $0.0 \%$ & $0.0 \%$ & $0.0 \%$ & $2.4 \%$ & $0.0 \%$ \\
\hline 2413 & $0.0 \%$ & $1.7 \%$ & $4.3 \%$ & $0.0 \%$ & $0.0 \%$ & $0.0 \%$ & $4.0 \%$ & $0.0 \%$ & $0.0 \%$ & $0.0 \%$ \\
\hline 2431 & $0.0 \%$ & $0.0 \%$ & $0.0 \%$ & $0.0 \%$ & $0.0 \%$ & $3.1 \%$ & $0.0 \%$ & $0.0 \%$ & $0.0 \%$ & $0.0 \%$ \\
\hline 3124 & $1.9 \%$ & $3.4 \%$ & $4.3 \%$ & $2.8 \%$ & $7.7 \%$ & $0.0 \%$ & $8.0 \%$ & $0.0 \%$ & $2.4 \%$ & $0.0 \%$ \\
\hline 3142 & $0.0 \%$ & $0.0 \%$ & $0.0 \%$ & $0.0 \%$ & $0.0 \%$ & $0.0 \%$ & $0.0 \%$ & $0.0 \%$ & $0.0 \%$ & $0.0 \%$ \\
\hline 3214 & $11.3 \%$ & $8.6 \%$ & $12.8 \%$ & $8.3 \%$ & $5.1 \%$ & $0.0 \%$ & $0.0 \%$ & $3.1 \%$ & $0.0 \%$ & $0.0 \%$ \\
\hline 3241 & $0.0 \%$ & $0.0 \%$ & $2.1 \%$ & $0.0 \%$ & $0.0 \%$ & $0.0 \%$ & $2.0 \%$ & $0.0 \%$ & $0.0 \%$ & $0.0 \%$ \\
\hline 3412 & $0.0 \%$ & $0.0 \%$ & $2.1 \%$ & $0.0 \%$ & $5.1 \%$ & $0.0 \%$ & $0.0 \%$ & $0.0 \%$ & $0.0 \%$ & $0.0 \%$ \\
\hline 3421 & $5.7 \%$ & $3.4 \%$ & $0.0 \%$ & $0.0 \%$ & $0.0 \%$ & $0.0 \%$ & $0.0 \%$ & $0.0 \%$ & $0.0 \%$ & $0.0 \%$ \\
\hline 4123 & $1.9 \%$ & $3.4 \%$ & $2.1 \%$ & $0.0 \%$ & $2.6 \%$ & $6.3 \%$ & $2.0 \%$ & $0.0 \%$ & $0.0 \%$ & $2.0 \%$ \\
\hline 4132 & $0.0 \%$ & $1.7 \%$ & $0.0 \%$ & $0.0 \%$ & $0.0 \%$ & $0.0 \%$ & $0.0 \%$ & $0.0 \%$ & $0.0 \%$ & $0.0 \%$ \\
\hline 4213 & $1.9 \%$ & $1.7 \%$ & $0.0 \%$ & $2.8 \%$ & $7.7 \%$ & $3.1 \%$ & $2.0 \%$ & $0.0 \%$ & $0.0 \%$ & $0.0 \%$ \\
\hline 4231 & $1.9 \%$ & $3.4 \%$ & $4.3 \%$ & $0.0 \%$ & $0.0 \%$ & $0.0 \%$ & $0.0 \%$ & $3.1 \%$ & $0.0 \%$ & $0.0 \%$ \\
\hline 4312 & $0.0 \%$ & $6.9 \%$ & $8.5 \%$ & $8.3 \%$ & $0.0 \%$ & $0.0 \%$ & $0.0 \%$ & $0.0 \%$ & $0.0 \%$ & $2.0 \%$ \\
\hline 4321 & $30.2 \%$ & $12.1 \%$ & $6.4 \%$ & $8.3 \%$ & $2.6 \%$ & $6.3 \%$ & $2.0 \%$ & $0.0 \%$ & $4.8 \%$ & $0.0 \%$ \\
\hline Total & $100.0 \%$ & $100.0 \%$ & $100.0 \%$ & $100.0 \%$ & $100.0 \%$ & $100.0 \%$ & $100.0 \%$ & $100.0 \%$ & $100.0 \%$ & $100.0 \%$ \\
\hline$N$ & 53 & 58 & 47 & 36 & 39 & 32 & 50 & 32 & 42 & 51 \\
\hline
\end{tabular}

Note: Share of decisions as a percentage of choice situations with the same priority score. 
Table C.5: Empirical Distribution of Lists (One Shot)

\begin{tabular}{|c|c|c|c|c|c|c|c|c|c|c|}
\hline \multirow[t]{2}{*}{$\mathrm{ROL}$} & \multicolumn{10}{|c|}{ Priority Score } \\
\hline & 1 & 2 & 3 & 4 & 5 & 6 & 7 & 8 & 9 & 10 \\
\hline 1234 & $92.3 \%$ & $26.7 \%$ & $69.6 \%$ & $47.8 \%$ & $71.4 \%$ & $47.1 \%$ & $55.0 \%$ & $87.5 \%$ & $81.8 \%$ & $81.0 \%$ \\
\hline 1243 & $0.0 \%$ & $0.0 \%$ & $0.0 \%$ & $0.0 \%$ & $0.0 \%$ & $0.0 \%$ & $5.0 \%$ & $0.0 \%$ & $0.0 \%$ & $0.0 \%$ \\
\hline 1324 & $0.0 \%$ & $0.0 \%$ & $0.0 \%$ & $0.0 \%$ & $0.0 \%$ & $5.9 \%$ & $0.0 \%$ & $0.0 \%$ & $0.0 \%$ & $9.5 \%$ \\
\hline 1342 & $0.0 \%$ & $0.0 \%$ & $0.0 \%$ & $0.0 \%$ & $0.0 \%$ & $0.0 \%$ & $0.0 \%$ & $0.0 \%$ & $0.0 \%$ & $0.0 \%$ \\
\hline 1423 & $0.0 \%$ & $0.0 \%$ & $0.0 \%$ & $0.0 \%$ & $0.0 \%$ & $0.0 \%$ & $0.0 \%$ & $0.0 \%$ & $0.0 \%$ & $0.0 \%$ \\
\hline 1432 & $0.0 \%$ & $0.0 \%$ & $0.0 \%$ & $0.0 \%$ & $0.0 \%$ & $0.0 \%$ & $0.0 \%$ & $0.0 \%$ & $0.0 \%$ & $0.0 \%$ \\
\hline 2134 & $0.0 \%$ & $20.0 \%$ & $8.7 \%$ & $13.0 \%$ & $14.3 \%$ & $17.6 \%$ & $20.0 \%$ & $4.2 \%$ & $4.5 \%$ & $0.0 \%$ \\
\hline 2143 & $0.0 \%$ & $0.0 \%$ & $0.0 \%$ & $0.0 \%$ & $0.0 \%$ & $0.0 \%$ & $5.0 \%$ & $0.0 \%$ & $0.0 \%$ & $0.0 \%$ \\
\hline 2314 & $0.0 \%$ & $6.7 \%$ & $0.0 \%$ & $4.3 \%$ & $0.0 \%$ & $5.9 \%$ & $5.0 \%$ & $0.0 \%$ & $0.0 \%$ & $0.0 \%$ \\
\hline 2341 & $7.7 \%$ & $6.7 \%$ & $0.0 \%$ & $4.3 \%$ & $7.1 \%$ & $0.0 \%$ & $0.0 \%$ & $0.0 \%$ & $0.0 \%$ & $0.0 \%$ \\
\hline 2413 & $0.0 \%$ & $0.0 \%$ & $0.0 \%$ & $4.3 \%$ & $0.0 \%$ & $0.0 \%$ & $0.0 \%$ & $0.0 \%$ & $4.5 \%$ & $0.0 \%$ \\
\hline 2431 & $0.0 \%$ & $0.0 \%$ & $0.0 \%$ & $0.0 \%$ & $0.0 \%$ & $0.0 \%$ & $0.0 \%$ & $0.0 \%$ & $0.0 \%$ & $0.0 \%$ \\
\hline 3124 & $0.0 \%$ & $0.0 \%$ & $8.7 \%$ & $0.0 \%$ & $0.0 \%$ & $5.9 \%$ & $0.0 \%$ & $0.0 \%$ & $0.0 \%$ & $0.0 \%$ \\
\hline 3142 & $0.0 \%$ & $0.0 \%$ & $0.0 \%$ & $0.0 \%$ & $0.0 \%$ & $0.0 \%$ & $0.0 \%$ & $0.0 \%$ & $0.0 \%$ & $0.0 \%$ \\
\hline 3214 & $0.0 \%$ & $6.7 \%$ & $8.7 \%$ & $4.3 \%$ & $0.0 \%$ & $5.9 \%$ & $0.0 \%$ & $0.0 \%$ & $0.0 \%$ & $4.8 \%$ \\
\hline 3241 & $0.0 \%$ & $0.0 \%$ & $0.0 \%$ & $8.7 \%$ & $0.0 \%$ & $0.0 \%$ & $0.0 \%$ & $0.0 \%$ & $0.0 \%$ & $0.0 \%$ \\
\hline 3412 & $0.0 \%$ & $6.7 \%$ & $4.3 \%$ & $0.0 \%$ & $0.0 \%$ & $0.0 \%$ & $0.0 \%$ & $0.0 \%$ & $0.0 \%$ & $0.0 \%$ \\
\hline 3421 & $0.0 \%$ & $0.0 \%$ & $0.0 \%$ & $0.0 \%$ & $0.0 \%$ & $5.9 \%$ & $0.0 \%$ & $0.0 \%$ & $0.0 \%$ & $4.8 \%$ \\
\hline 4123 & $0.0 \%$ & $6.7 \%$ & $0.0 \%$ & $0.0 \%$ & $0.0 \%$ & $0.0 \%$ & $0.0 \%$ & $0.0 \%$ & $0.0 \%$ & $0.0 \%$ \\
\hline 4132 & $0.0 \%$ & $0.0 \%$ & $0.0 \%$ & $0.0 \%$ & $0.0 \%$ & $0.0 \%$ & $0.0 \%$ & $4.2 \%$ & $4.5 \%$ & $0.0 \%$ \\
\hline 4213 & $0.0 \%$ & $0.0 \%$ & $0.0 \%$ & $4.3 \%$ & $7.1 \%$ & $0.0 \%$ & $0.0 \%$ & $0.0 \%$ & $0.0 \%$ & $0.0 \%$ \\
\hline 4231 & $0.0 \%$ & $0.0 \%$ & $0.0 \%$ & $0.0 \%$ & $0.0 \%$ & $0.0 \%$ & $5.0 \%$ & $4.2 \%$ & $4.5 \%$ & $0.0 \%$ \\
\hline 4312 & $0.0 \%$ & $0.0 \%$ & $0.0 \%$ & $0.0 \%$ & $0.0 \%$ & $0.0 \%$ & $0.0 \%$ & $0.0 \%$ & $0.0 \%$ & $0.0 \%$ \\
\hline 4321 & $0.0 \%$ & $20.0 \%$ & $0.0 \%$ & $8.7 \%$ & $0.0 \%$ & $5.9 \%$ & $5.0 \%$ & $0.0 \%$ & $0.0 \%$ & $0.0 \%$ \\
\hline Total & $100.0 \%$ & $100.0 \%$ & $100.0 \%$ & $100.0 \%$ & $100.0 \%$ & $100.0 \%$ & $100.0 \%$ & $100.0 \%$ & $100.0 \%$ & $100.0 \%$ \\
\hline$N$ & 13 & 15 & 23 & 23 & 14 & 17 & 20 & 24 & 22 & 21 \\
\hline
\end{tabular}

Note: Share of decisions as a percentage of choice situations with the same priority score. 


\section{Face-Value Beliefs}

Table D.1: Mixed and Standard Logit Specifications

\begin{tabular}{|c|c|c|c|}
\hline & $\begin{array}{c}(1) \\
\text { Multiround } \\
\text { All } \\
\text { (Logit) }\end{array}$ & $\begin{array}{c}(2) \\
\text { One Shot } \\
\text { All } \\
\text { (Logit) }\end{array}$ & $\begin{array}{c}(3) \\
\text { Multiround } \\
\text { Misrepresenters Only } \\
\text { (Mixed Logit, Normal Dist.) }\end{array}$ \\
\hline$\alpha_{1}($ normalized $)$ & $\begin{array}{c}2 \\
(0.1)\end{array}$ & $\begin{array}{c}2 \\
(0.2)\end{array}$ & $\begin{array}{c}2 \\
(0.14)\end{array}$ \\
\hline$\lambda$ & $\begin{array}{c}1.76 \\
(0.18)\end{array}$ & $\begin{array}{c}1.63 \\
(0.37)\end{array}$ & \\
\hline$\mu_{\lambda}$ & & & $\begin{array}{c}2.42 \\
(0.45)\end{array}$ \\
\hline$\sigma_{\lambda}$ & & & $\begin{array}{c}2.31 \\
(0.37)\end{array}$ \\
\hline Log-likelihood & -1717.58 & -466.58 & -1154.66 \\
\hline Likelihood ratio test & 19.90 & 3.15 & \\
\hline$p$-value & 0.000 & 0.076 & \\
\hline$N$ & 720 & 192 & 440 \\
\hline
\end{tabular}

Notes: The parameters were estimated through Maximum Likelihood (columns 1 and 2) and Maximum Simulated Likelihood with 1,000 draws (column 3). The probability of winning each prize is based on correct beliefs about priorities, assuming other players play truthfully. Likelihood ratio test: $\mathrm{df}=1, H_{0}: \lambda=1$. 


\section{E Early vs Later Rounds}

Figure E.1: Empirical Distribution of Lists (by Five Rounds)

(a) First Five Rounds
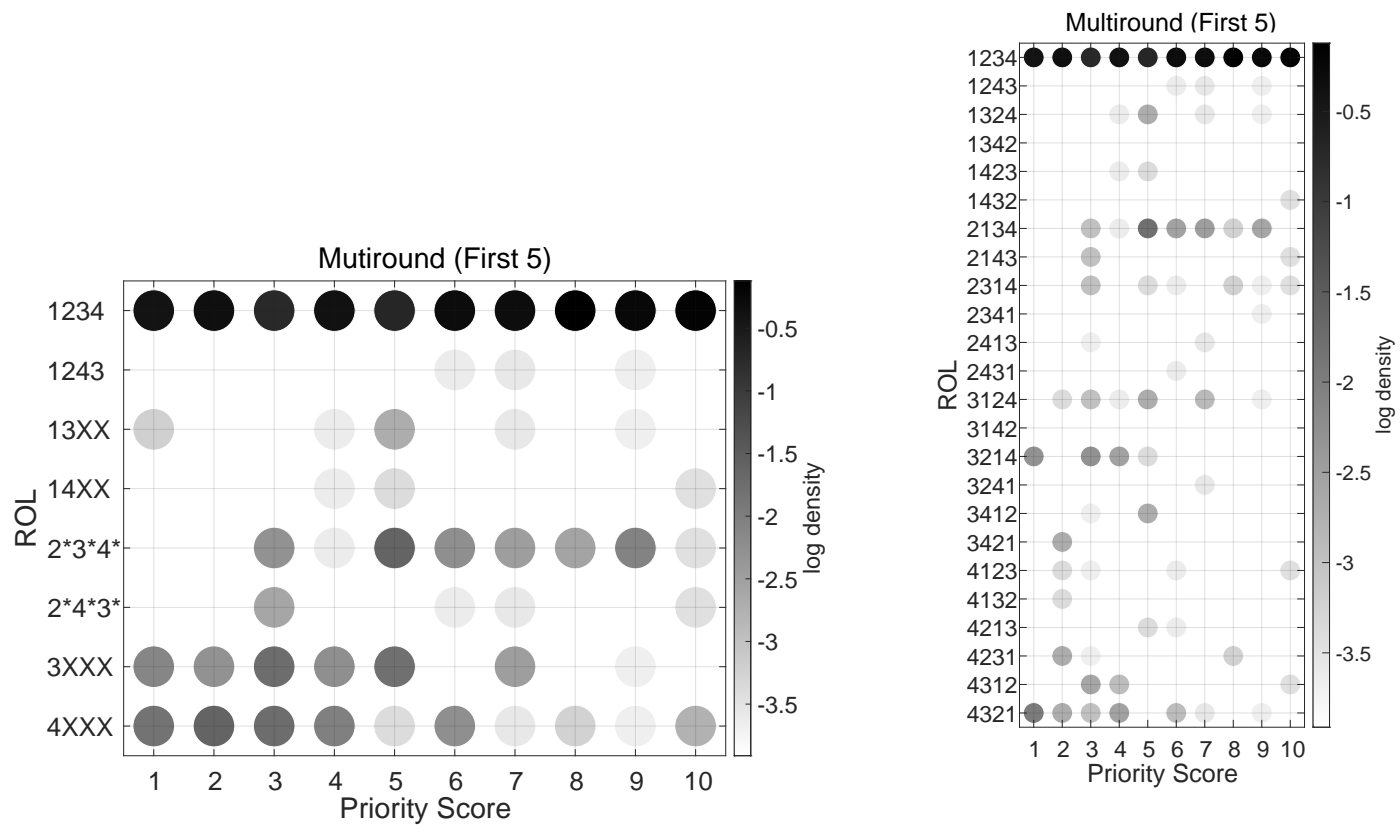

(b) Last Five Rounds
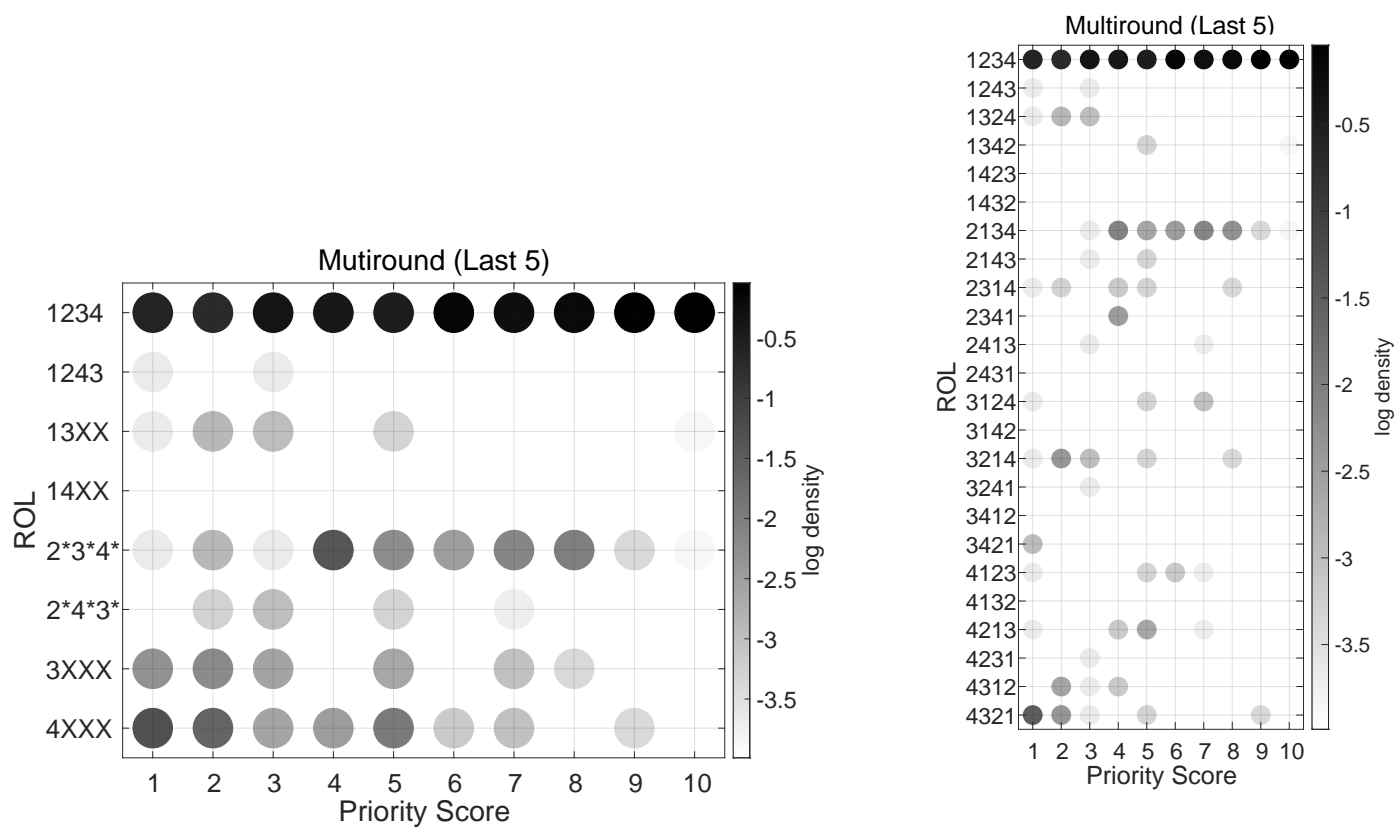

Notes: $N=360 . \log$ density as a share of observations with the same priority score. Panel (a): ROLs are grouped as explained in figure $4 \mathrm{a}$. 
Table E.1: Standard Logit (Earlier vs Later Rounds)

\begin{tabular}{lcc}
\hline \hline & $(1)$ & $(2)$ \\
& Multiround & Multiround \\
& First 5 Rounds & Last 5 Rounds \\
& $($ Logit) & (Logit) \\
$\alpha_{1}$ (normalized) & 2 & 2 \\
& $(0.14)$ & $(0.12)$ \\
$\lambda$ & & \\
& 1.86 & 2.10 \\
Log-likelihood & $(0.28)$ & $(0.24)$ \\
Likelihood ratio test & & -766.17 \\
$p$-value & -877.67 & 24.64 \\
$N$ & 10.37 & 0.000 \\
\hline
\end{tabular}

Notes: The parameters were estimated through Maximum Likelihood. The probability of winning each prize is the simulated probability as described in 3.2 Likelihood ratio test: $\mathrm{df}=1, H_{0}: \lambda=1$. 